\title{
Frequency Offset Estimation for MB-OFDM-based UWB Systems
}

\author{
Yinghui Li, Student Member, IEEE, Hlaing Minn, Senior Member, IEEE,, Trent Jacobs, Student Member, IEEE, \\ and Moe Z. Win, Fellow, IEEE
}

\begin{abstract}
We address low-complexity, highly-accurate frequency offset estimation for multi-band orthogonal frequency division multiplexing (MB-OFDM) based ultra-wide band systems in time-invariant as well as time-variant channels. We investigate the unique characteristics of MB-OFDM systems, namely, different frequency offsets, channel responses, received energies, and preamble structures in different frequency bands. Utilizing them, we develop frequency offset estimators based on the best linear unbiased estimation principle. If compared to the reference estimators, our proposed methods achieve significantly better estimation performance ( 4 to $6.4 \mathrm{~dB}(5$ to $20 \mathrm{~dB}$ ) estimation mean-square error advantage in the time-invariant (time-variant) channels) for all preamble patterns of the MB-OFDM system in [8].
\end{abstract}

Index Terms-BLUE, frequency offset, OFDM, time-variant channel, UWB.

\section{INTRODUCTION}

$\mathbf{U}$ LTRA-WIDE band (UWB) systems (e.g., impulse radios [1] [2] and transmitted reference schemes [3] to name a few) offer improved ranging precision, better penetration through obstacles, higher data rate, and increased multipath or frequency diversity. Due to ultra-wide bandwidth, UWB systems can operate at low power spectral density which allows overlay with other narrow-band systems. The coexistence issue and the spectral analysis of UWB systems have been investigated in [4]- [6]. Another approach to realize UWB systems is multi-band orthogonal frequency division multiplexing (MB-OFDM) [7] which has been proposed for the IEEE 802.15.3a standard [8] and adopted in European Computer Manufacturers Association (ECMA) standard [9]. The very high data rate (480 Mbps and beyond) capability of the UWB technology would provide a compelling cable-replacement wireless technology. OFDM is a relatively mature technology and has been adopted in digital broadcasting, wireless LAN and MAN standards [10]- [13]. OFDM has several advantages such as low complexity equalization in dispersive channels and the spectral density scalability/adaptability (e.g., adaptive bit loading [14]). However, OFDM has some disadvantages such

Paper approved by C. Tepedelenlioglu, the Editor for Synchronization and Equalization of the IEEE Communications Society. Manuscript received July 8, 2006; revised December 18, 2006.

Y. Li, H. Minn, and T. Jacobs are with the Department of Electrical Engineering, University of Texas at Dallas, P.O. Box 830688, EC 33, Richardson, TX 75080 USA (e-mail: \{yx1044000, hlaing.minn, taj016000\}@ utdallas.edu).

M. Z. Win is with the Laboratory for Information and Decision Systems (LIDS), Massachusetts Institute of Technology (MIT), 77 Massachusetts Avenue, Room 32-D658, Cambridge, MA 02139 USA (e-mail: moewin@mit.edu).

Digital Object Identifier 10.1109/TCOMM.2008.060343. as larger susceptibility to nonlinear distortion at the transmit power amplifier [15] and larger sensitivity to frequency offsets [16]- [19]. The application of OFDM technology in UWB systems demands highly accurate frequency offset estimation, since frequency offset causes a loss of orthogonality among the subcarriers thereby introducing inter sub-carrier interference and significantly degrading the error performance.

In the MB-OFDM system in [8] and [9], a preamble is used to aid receiver algorithms related to timing and frequency synchronization, and channel estimation. There are several preamble-based frequency offset estimators (e.g., [20]- [26]) in the research literature. However, we have not observed frequency offset estimators derived for the MB-OFDM systems. In MB-OFDM systems, the oscillator frequency offset (OFO) causes different carrier frequency offsets (CFOs) for the different bands. The channel frequency responses and the channel energies for the different bands are different as well. The frequency hopping of the MB-OFDM system results in different preamble structures for some of the frequency bands. These characteristics neither exist in typical OFDM systems nor have been fully reported in the MB-OFDM literature. To our best knowledge, no frequency offset estimator has been developed utilizing all of the above characteristics of MBOFDM systems for performance improvement.

The statistics of UWB channels reported in the literature are mainly time-invariant (see [27]- [33]). However, moving scatterers can cause time-varying UWB channels [34] [35]. The typical channel variations due to moving persons in UWB applications would be very slow and their effects on frequency offset estimation are negligible. However, occasionally there may exist very fast moving scatterers which cause a sudden ${ }^{1}$ change in the channel and degrade the frequency offset estimation in UWB systems (e.g., control, monitoring, and other electronic transaction systems for high speed vehicles). To our best knowledge, there is no frequency offset estimator designed to be robust against sudden channel changes.

In this paper, we characterize the unique features of MBOFDM systems. Using a simple channel model capturing occasional abrupt channel change behavior, we develop an efficient detection algorithm to track a sudden channel change and propose frequency offset estimators suitable for (sudden) time-variant as well as time-invariant UWB systems. We apply the best linear unbiased estimation (BLUE) principle and fully utilize the unique characteristics of the MB-OFDM

\footnotetext{
${ }^{1}$ It is a relative term and here we mean the change duration is about one symbol interval or less.
} 


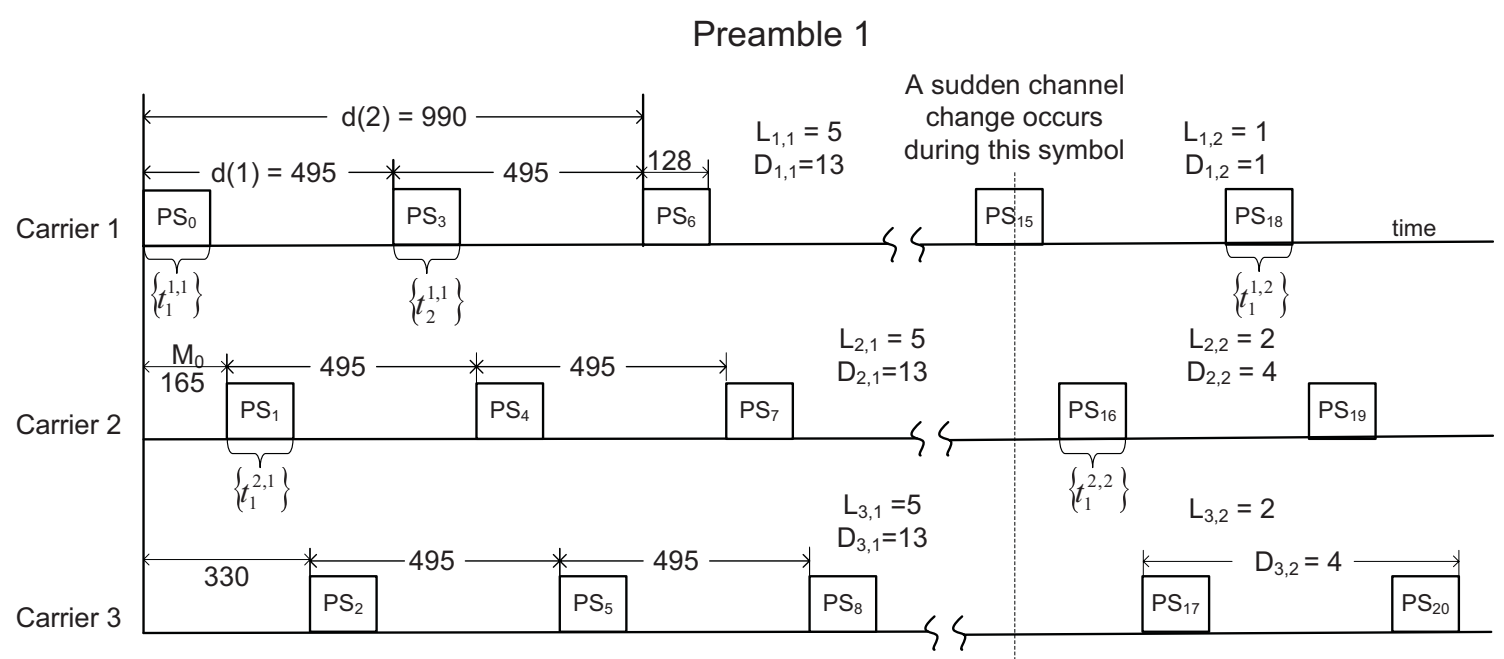

Fig. 1. An illustration of the preamble structure and the related parameters of the proposed estimators for the preamble pattern 1 (or 2) from [8].

systems in developing our frequency offset estimators. Our proposed estimators offer significant MSE improvement over the reference estimators (about 4-6.4 dB (5-20 dB) estimation mean-square error (MSE) advantage in time-invariant (timevariant) UWB channels).

The rest of this paper is organized as follows. Section II describes the signal and channel models. The proposed frequency offset estimation methods are presented in Section III, and the abrupt channel change detection algorithm is described in Section IV. Section V discusses the design parameters. Simulation results and discussions are provided in Section VI and the paper is concluded in Section VII.

\section{Signal And Channel Models}

In the MB-OFDM-based UWB system [8], the carrier frequency is hopped within a pre-defined set of carrier frequencies $\left\{f_{1}, f_{2}, f_{3}\right\}$ (corresponding to disjoint frequency bands) from symbol to symbol, according to a time-frequency code. The MB-OFDM system from [8] has 4 different preamble patterns (for 4 pico-nets), each associated with a different time-frequency code. Each preamble pattern is constructed by successively repeating a time-domain preamble sequence (symbol) over 21 periods as $\left\{\mathrm{PS}_{0}, \mathrm{PS}_{1}, \ldots, \mathrm{PS}_{20}\right\}$ (see Fig. 1). The preamble patterns 1 and 2 (or, 3 and 4 ) have the same structure, except for the ordering of the carrier frequencies. The preamble pattern 3 or 4 , however, has a structure distinct from pattern 1 or 2 . For details of the preamble structures of preamble 3 or 4, please see Fig. 2 in [36].

In the MB-OFDM-based system from [8], zero-padded guard intervals ( $N_{\text {pre }}$ prefix and $N_{\text {suf }}$ suffix zero samples; $N_{g}$ $\left.=N_{\text {pre }}+N_{\text {suf }}\right)$ are used instead of the conventional cyclic prefix guard interval. The total number of samples in one OFDM symbol (including the guard samples) is $M_{0}=N+N_{g}$. The low-pass-equivalent time-domain samples $\left\{s_{q}\left(k=m M_{0}+\right.\right.$ $\left.\left.N_{\text {pre }}+n\right): n=-N_{\text {pre }}, \ldots, N+N_{\text {suf }}-1\right\}$ (sampled at $N / T=1 / T_{s}, N$ times the sub-carrier spacing) transmitted during the $m$-th symbol interval in the $q$-th frequency band are generated by taking the $N$-point inverse discrete Fourier transform of the corresponding sub-carrier symbols $\left\{C_{q, m}(l)\right\}$ (zeros for null sub-carriers; all zeros if no symbols are transmitted in the $q$-th band during the $m$-th interval) and inserting zero guard-samples as ${ }^{2}$

$$
s_{q}(k)=\left\{\begin{array}{l}
\frac{1}{N} \sum_{l=0}^{N-1} C_{q, m}(l) e^{j 2 \pi l n / N}, 0 \leq n \leq N-1 \\
0,-N_{\text {pre }} \leq n<0 ; N \leq n<N+N_{\text {suf }}
\end{array}\right.
$$

The IEEE 802.15.3a UWB RF channel model described in [34] is given by

$$
h_{\mathrm{RF}}(t)=X \sum_{l=0}^{L_{h}} \sum_{k=0}^{K} \alpha_{k, l} \delta\left(t-T_{l}-\tau_{k, l}\right)
$$

where $\alpha_{k, l}, T_{l}, \tau_{k, l}$, and $X$ are random variables representing the multipath gain coefficients, the delay of the $l$-th cluster, the delay (relative to the $l$-th cluster arrival time) of the $k$-th multipath component of the $l$-th cluster, and the log-normal shadowing on the amplitude, respectively. $L_{h}+1$ is the number of the clusters and $K+1$ is the number of the multi-path components per cluster. Details of the channel models are referred to in [34]. In this paper, we consider a low-passequivalent system that absorbs the carrier-frequency hopping into the channel impulse response (CIR). The sample-spaced low-pass-equivalent CIR for the $q$-th band is given by

$$
h_{q}(n)=X \sum_{l=0}^{L_{h}} \sum_{k=0}^{K} \alpha_{k, l} e^{-j 2 \pi f_{q}\left(T_{l}+\tau_{k, l}\right)} p\left(n T-T_{l}-\tau_{k, l}-t_{0}\right)
$$

where the effect of the combined transmit and receive filter with the impulse response $p(t)$ has been included in the CIR and $t_{0}$ is a delay for the causality. From (3), we can observe that the channel responses and energies vary with frequency band.

The above UWB channel model does not include timevarying behavior. However, the movements of the transmitter, the receiver, and/or the scatterers result in a time-varying channel [34] [35]. For the UWB system in [8], the channel variations due to the Doppler spread are too slow and can be neglected in the $\mathrm{CFO}$ estimation. However, occasionally when a fast scatterer moves through and thus blocks one or more

\footnotetext{
${ }^{2}$ Note that for each band, we use the corresponding low-pass-equivalent system. The effects of different carrier frequencies are embedded in the lowpass-equivalent channels.
} 


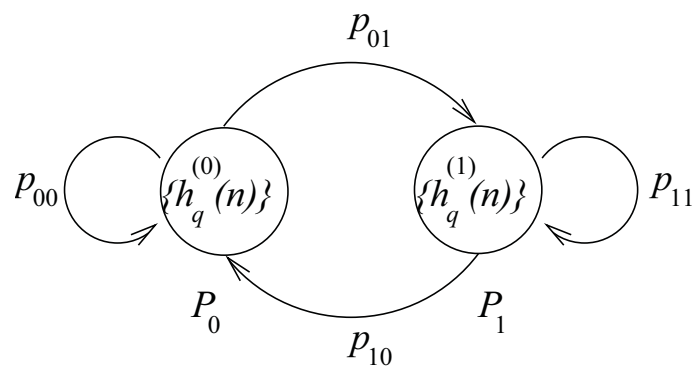

Fig. 2. A simple time-variant UWB channel model capturing occasional sudden channel changes.

of the UWB channel paths, an abrupt change in the CIR will occur. A scatterer can move into or out of a position that blocks some channel paths. We consider that the paths affected by the fast moving scatterer correspond to the $L_{c}$-th cluster, which is a random variable. For convenience, we assume that arbitrary $\mathcal{J}$ adjacent rays of the $L_{c}$-th cluster are completely blocked. Note that $0 \leq \mathcal{J} \leq K$ and $\mathcal{J}=0$ corresponds to a timeinvariant channel while $\mathcal{J}=K$ with $k_{0}=0$ defines that the whole $L_{c}$-th cluster is blocked. Due to non-negligible physical sizes of moving scatters in typical UWB environments, after an abrupt change occurs, the channel will most likely remain the same for the rest of the packet. The corresponding timevariant channel during the preamble transmission can then be modeled as a two state Markov process as shown in Fig. 2. The channel model in state zero, $\left\{h_{q}^{(0)}(n)\right\}$, represents the UWB channel without any blocking of the channel paths. The channel model in state one, $\left\{h_{q}^{(1)}(n)\right\}$, represents the case in which some channel paths are blocked by a fast moving scatterer. The discrete-time low-pass-equivalent CIRs of the two states are given by

$$
\begin{aligned}
& h_{q}^{(0)}(n)=X \sum_{l=0}^{L_{h}} \sum_{k=0}^{K} \alpha_{k, l} e^{-j 2 \pi f_{q}\left(T_{l}+\tau_{k, l}\right)} p\left(n T-T_{l}-\tau_{k, l}-t_{0}\right) \\
& h_{q}^{(1)}(n)=h_{q}^{(0)}(n) \\
& \quad-X\left[\sum_{k=k_{0}}^{k_{0}+\mathcal{J}-1} \alpha_{k, l} e^{-j 2 \pi f_{q}\left(T_{l}+\tau_{k, l}\right)} p\left(n T-T_{l}-\tau_{k, l}-t_{0}\right)\right]_{l=L_{c}}
\end{aligned}
$$

where $L_{c}$ is uniformly distributed with probability $P\left(L_{c}=\right.$ $i)=1 /\left(L_{h}+1\right), i=0,1 \ldots, L_{h}, k_{0}$ is also uniformly distributed over the integer range of $[0, K-\mathcal{J}+1]$, and $\mathcal{J}$ is a user-defined parameter. The state-transition probability matrix $^{3}$ is $\boldsymbol{P}=\left[p_{00}, p_{01} ; p_{10}, p_{11}\right]$ and the steady-state probability vector is $\left[P_{0}, P_{1}\right]^{T}$. The values of these probabilities depend on the environment. Since an abrupt channel change can occur at any time randomly, it is most reasonable to assume that if there is an abrupt channel change during the preamble transmission, the time instant of the abrupt change is uniformly distributed over the preamble duration (21 periods). When $P_{0}=1$ and $p_{00}=1$, the channel model represents the time-invariant channel.

For the mandatory Mode- 1 of the MB-OFDM system in [7] [8], the three carrier frequencies are generated from one oscillator (see [36] [38]). The normalized (by the sub-carrier spacing) OFO $v$ causes different normalized CFOs $\left\{v_{q}\right\}$ for

${ }^{3}$ We use MATLAB notation in representing a matrix. the three bands, and their relationship ${ }^{4}$ is given by

$$
\begin{aligned}
& v_{q}=b_{q} v, \text { for } q=1,2,3 \\
& {\left[b_{1}, b_{2}, b_{3}\right]=\left[\frac{13}{16}, \frac{15}{16}, \frac{17}{16}\right] .}
\end{aligned}
$$

We assume that the timing synchronization eliminates intersymbol-interference. Define $\left\{t_{l}^{q}\right\}=\left\{t_{l}^{q}(i): i=0, \ldots, N-\right.$ $\left.1 ; l=1, \ldots, L_{q}\right\}$ where $\left\{t_{l}^{q}(i): i=0,1, \ldots, N-1\right\}$ denotes the time-domain sample index set corresponding to the $l$-th non-zero preamble symbol period in the $q$-th band. $L_{q}$ is the number of nonzero preamble symbols in the $q$-th band and depends on the preamble pattern and band index $q$ (c.f. Fig. 2 in [36]). Let $\left\{x_{q}(k)\right\}$ denote the low-pass-equivalent timedomain channel output (noise-free) signal samples corresponding to the $q$-th band, where $x_{q}(k) \triangleq x_{q}\left(t_{0}+k T_{s}\right)$. Then the corresponding low-pass-equivalent time-domain received samples $\left\{r_{q}\left(t_{l}^{q}(i)\right)\right\}$, where $r_{q}(k) \triangleq r_{q}\left(t_{0}+k T_{s}\right)$, in the $q$-th band can be expressed as

$$
r_{q}\left(t_{l}^{q}(i)\right)=e^{j \varphi} e^{j 2 \pi v_{q} t_{l}^{q}(i) / N} x_{q}\left(t_{l}^{q}(i)\right)+n\left(t_{l}^{q}(i)\right)
$$

where $v_{q}$ is the normalized CFO of the $q$-th band, $\varphi$ is an arbitrary carrier phase, $\left\{n\left(t_{l}^{q}(i)\right)\right\}$ are independent and identically-distributed, circularly-symmetric complex Gaussian noise samples with zero mean and variance $\sigma^{2}=$ $E\left\{\left|n\left(t_{l}^{q}(i)\right)\right|^{2}\right\}$.

\section{Proposed Frequency Offset Estimation}

As discussed in the previous section, UWB systems may undergo abrupt channel changes. When an abrupt channel change occurs in the middle of the preamble transmission, the CFO estimation can be significantly affected due to the coherence loss in the periodicity of the received preamble symbols. In this section, we develop a BLUE-based OFO estimator for time-invariant/time-variant UWB channels. The novelty of the proposed approach compared to existing BLUEbased approaches is the incorporation of the MB OFDM characteristics in the estimator development.

For time-variant UWB channels, we first split the preamble in each band into two ${ }^{5}$ parts (left and right) by a simple abrupt channel change detection algorithm, which will be described in Section IV. We denote these parts by $p$-th preamble part with $p=1$ and 2 for the left and right part, respectively. The underlying idea is that correlation-based estimators require identical channel output preamble symbols, and due to the abrupt change, the channel outputs corresponding to these two parts will not be identical, and hence should not be crosscorrelated. Next we estimate CFO based on each part, and then appropriately average the CFO estimates.

For time-invariant UWB channels, since they are the special cases of time-variant channels with the steady-state probability vector $[1,0]^{T}$, we can apply the method developed for the time-variant channel except with the following changes: we skip the abrupt channel change detection step, set the left part of the preamble in each band to be the preamble itself and hence null the right part.

\footnotetext{
${ }^{4}$ Our proposed estimator can be applied to other implementations of carrier frequencies generation by changing the values in (7) accordingly.

${ }^{5}$ Due to non-negligible physical sizes of moving scatters, the channel change occurs only once (if it does) during the preamble transmission, and hence we just need to consider two parts.
} 


\section{A. OFO Estimation}

The proposed OFO estimator is based on the BLUE principle and the correlation among the non-zero received preamble symbols within the same part of the band. For every preamble pattern, the channel output preamble of each part in a single band has identical symbols. The channel output preambles of different preamble parts are not the same due to distinct channel responses.

Let $D_{q, p}$ denote the total number of periods counting from the first non-zero preamble period of the $p$-th preamble part to the end of the last non-zero preamble period of the $p$ th preamble part in the $q$-th band. Then $N_{q, p}=D_{q, p} M_{0}$ represents the number of samples of $D_{q, p}$ periods where $M_{0}=N+N_{g}$ as defined before. Let $\left\{t_{l}^{q, p}\right\}$ denote the nonzero sample index set for the $p$-th preamble part in the $q$-th band (c.f. Fig. 1). Define the correlation term with a correlation distance $d(m)$ between non-zero received symbols of the $p$-th preamble part in the $q$-th band as

$$
R_{q, p}(m)=\sum_{\{k, k+d(m)\} \in\left\{t_{l}^{q, p}\right\}} r_{q}^{*}(k) r_{q}(k+d(m))
$$

where $1 \leq m \leq H_{q, p}, H_{q, p}$ is a design parameter with $M_{0} \leq d\left(H_{q, p}\right) \leq N_{q, p}$, and $d(m), m=1,2, \ldots$, is an integer multiple of $M_{0}$. For the preamble pattern 1 or $2, d(m)=$ $3 m M_{0}$ in all the three frequency bands, and for the preamble pattern 3 or $4, d(m)=6 m M_{0},(6(m-1)+1) M_{0}$, or $(6 m-$ 1) $M_{0}$. By substituting (8) into (9), we obtain

$$
R_{q_{\_} p}(m)=e^{\frac{j 2 \pi v_{q} d(m)}{N}}\left\{Q_{q_{\_} p}(m) E_{q_{\_} p}+G_{q_{\_} p}(m)+\mathcal{N}_{q_{\_} p}(m)\right\}
$$

where

$$
\begin{aligned}
& Q_{q_{-} p}(m) \triangleq\left\lfloor\frac{N_{q_{-} p}-d(m)-M_{0}}{d_{0}}\right\rfloor+1 \\
& d_{0}= \begin{cases}3 M_{0}, & \text { preamble } 1 \& 2 \\
6 M_{0}, & \text { preamble } 3 \& 4\end{cases} \\
& E_{q_{-} p} \triangleq \sum_{k=0}^{N-1}\left|x_{q}\left(t_{l}^{q_{-} p}(k)\right)\right|^{2} \\
& G_{q_{-} p}(m) \triangleq \sum_{\{k, k+d(m)\} \in\left\{t_{-}^{q_{-} p^{p}}\right\}}\left\{x_{q}^{*}(k) \widetilde{n}(k+d(m))\right. \\
& \left.+x_{q}(k+d(m)) \widetilde{n}^{*}(k)\right\} \\
& \mathcal{N}_{q_{-} p}(m) \triangleq \sum_{\{k, k+d(m)\} \in\left\{t_{l}^{q_{-}}\right\}} \tilde{n}^{*}(k) \widetilde{n}(k+d(m))
\end{aligned}
$$

and $\lfloor\cdot\rfloor$ denotes the floor operation. Note that $\widetilde{n}(k) \triangleq$ $n(k) e^{-j 2 \pi v k / N}$ is statistically equivalent to $n(k)$. Define

$$
\theta_{q, p}(m) \triangleq \frac{N}{2 \pi d(m)} \text { angle }\left\{R_{q, p}(m)\right\} .
$$

If $\left|v_{q}\right|<N /(2 d(m))$, then we have

$$
\begin{aligned}
& \theta_{q_{\_} p}(m)=v_{q} \\
& \quad+\frac{N}{2 \pi d(m)} \text { angle }\left\{Q_{q_{-} p}(m) E_{q_{\_} p}+G_{q_{-} p}(m)+\mathcal{N}_{q_{\_} p}(m)\right\}
\end{aligned}
$$

which gives an estimate of $v_{q}$. The CFO estimator for $v_{q}$ based on the BLUE principle [39] using the $p$-th preamble part in the $q$-th band can then be given by

$$
\widehat{v}_{q, p}=\sum_{m=1}^{H_{q, p}} \omega_{q, p}(m) \theta_{q, p}(m)
$$

where $\omega_{q, p}(m)$ is the $m$-th component of the weighting vector

$$
\boldsymbol{\omega}_{q, p}=C_{\theta_{q, p}}^{-1} \mathbf{1}\left(\mathbf{1}^{T} C_{\theta_{q, p}}^{-1} \mathbf{1}\right)^{-1} .
$$

In (19), $\boldsymbol{C}_{\theta_{q, p}}$ is the covariance matrix of $\left\{\theta_{q, p}(m): m=\right.$ $\left.1, \ldots, H_{q, p}\right\}$ and 1 denotes the all-one column vector, whose size should be apparent from the expression. Next, we combine the CFO estimates $\left\{\widehat{v}_{q, p}\right\}$ obtained from the different preamble parts in the various frequency bands by using the BLUE principle, together with (6) and (16), and we obtain the estimate of $v$ as

$$
\widehat{v}=\sum_{p=1}^{2} \sum_{q=1}^{3} \sum_{m=1}^{H_{q, p}} \bar{\omega}_{q, p}(m) \theta_{q, p} / b_{q}
$$

where the weighting values are given by

$$
\begin{aligned}
\overline{\boldsymbol{\omega}} & \triangleq\left[\bar{\omega}_{1 \_1}(1), \ldots, \bar{\omega}_{1 \__{2}}\left(H_{1 \_}\right), \ldots, \bar{\omega}_{3 \_1}(1), \ldots, \bar{\omega}_{3_{-} 2}\left(H_{3_{-} 2}\right)\right]^{T} \\
& =\boldsymbol{C}^{-1} \mathbf{1}\left(\mathbf{1}^{T} \boldsymbol{C}^{-1} \mathbf{1}\right)^{-1}
\end{aligned}
$$

and $\boldsymbol{C}$ is the covariance matrix of $\left[\theta_{1,1}(1) / b_{1}, \ldots\right.$, $\theta_{1,2}\left(H_{1,2}\right) / b_{1}, \theta_{2,1}(1) / b_{2}, \ldots, \theta_{2,2}\left(H_{2,2}\right) / b_{2}, \theta_{3,1}(1) / b_{3}, \ldots$, $\left.\theta_{3,2}\left(H_{3,2}\right) / b_{3}\right]$ given by

$$
\boldsymbol{C}=\operatorname{diag}\left\{\frac{\boldsymbol{C}_{\theta_{1,1}}}{b_{1}^{2}}, \frac{\boldsymbol{C}_{\theta_{1,2}}}{b_{1}^{2}}, \frac{\boldsymbol{C}_{\theta_{2,1}}}{b_{2}^{2}}, \frac{\boldsymbol{C}_{\theta_{2,2}}}{b_{2}^{2}}, \frac{\boldsymbol{C}_{\theta_{3,1}}}{b_{3}^{2}}, \frac{\boldsymbol{C}_{\theta_{3,2}}}{b_{3}^{2}}\right\} .
$$

From (18)-(22), we çan alşo express (20) as

$$
\widehat{v}=\frac{\sum_{p=1}^{2} \sum_{q=1}^{3} \mathbf{1}^{2} \boldsymbol{C}_{\theta_{q, p}}^{-1} b_{q} \widehat{v}_{q, p}}{\sum_{p=1}^{2} \sum_{q=1}^{3} \mathbf{1}^{T} \boldsymbol{C}_{\theta_{q, p}}^{-1} \mathbf{1} b_{q}^{2}} .
$$

In the following, by using two high SNR approximations of (17), we will present two methods for obtaining the covariance matrices required in the above estimation.

\section{B. Method A}

Equation (17) can be expressed as

$$
\theta_{q_{\_} p}(m)=v_{q}+\frac{N}{2 \pi d(m)} \tan ^{-1}\left(B_{q_{-} p}(m)\right)
$$

where

$$
B_{q_{\_} p}(m)=\frac{\Im\left\{G_{q_{\_} p}(m)\right\}+\Im\left\{\mathcal{N}_{q_{\_} p}(m)\right\}}{Q_{q_{\_} p}(m) E_{q_{\_} p}+\Re\left\{G_{q_{\_} p}(m)\right\}+\Re\left\{\mathcal{N}_{q_{-} p}(m)\right\}}
$$

$\Re\{X\}$ and $\Im\{X\}$ are the real and the imaginary parts of $X$, respectively, and $\tan ^{-1}$ is the four-quadrant arctangent function. For high SNR, we can approximate (24) as

$$
\theta_{q, p}(m) \simeq v_{q}+\frac{N}{2 \pi d(m)} \frac{\Im\left\{G_{q, p}(m)\right\}+\Im\left\{\mathcal{N}_{q, p}(m)\right\}}{Q_{q, p}(m) E_{q, p}} .
$$

Using (26), we derive the covariance matrices required in (23) for all the preamble patterns.

1) Preamble Patterns 1 \& 2: For the patterns 1 and 2, the preamble structure in each band is the same and all adjacent identical symbols have a distance of $3 M_{0}$. Thus $d(m)=3 m M_{0}$ and $Q_{q, p}(m)=\left(L_{q, p}-m\right)$ where $L_{q, p}$ is the number of non-zero identical symbols of the $p$-th part in the $q$-th band (see Fig. 1). After straightforward calculation, the $m$-th row, $n$-th column element of $\boldsymbol{C}_{\theta_{q, p}}$ for the preamble patterns 1 and 2 can be expressed as

$$
\begin{aligned}
& \boldsymbol{C}_{\theta_{q_{-} p}}(m, n)=\frac{N^{2} \sigma^{2}}{4 \pi^{2}\left(3 M_{0}\right)^{2} E_{q_{-} p}} \frac{1}{m n\left(L_{q_{-} p}-m\right)\left(L_{q_{-} p}-n\right)} \\
& \times \begin{cases}m+\frac{\left(L_{q_{\_} p}-m\right) N \sigma^{2}}{2 E_{q_{\_} p}}, & \text { if } m=n \& m<L_{q_{-} p} / 2 \\
\left(L_{q_{\_} p}-m\right)+\frac{\left(L_{q_{\_} p}-m\right) N \sigma^{2}}{2 E_{q_{\_} p}}, & \text { if } m=n \& m \geq L_{q_{\_} p} / 2 \\
\min (m, n), & \text { if } m \neq n \& m+n<L_{q_{-} p} \\
L_{q_{\_} p}-\max (m, n), & \text { if } m \neq n \& m+n \geq L_{q_{\_} p}\end{cases}
\end{aligned}
$$




$$
\begin{aligned}
& \boldsymbol{C}_{\theta_{q_{-} p}}^{(1,1)}(m, n)=\frac{N^{2} \sigma^{2}}{4 \pi^{2} E_{q_{-} p}} \frac{1}{d_{1}(m) d_{1}(n) Q_{q_{-} p, 1}(m) Q_{q_{-} p, 1}(n)}
\end{aligned}
$$

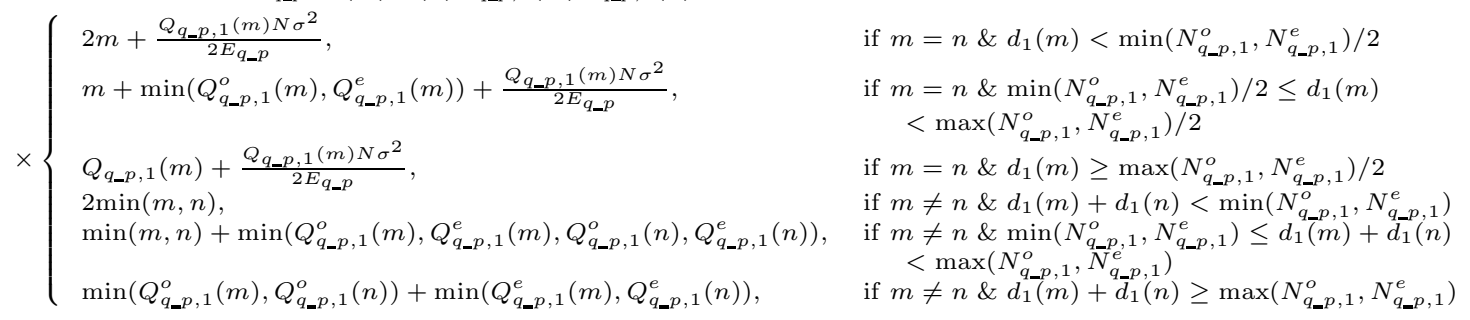

2) Preamble Patterns 3 \& 4: All possible correlation distances for the patterns 3 and 4 in all three bands are $\left\{M_{0}, 5 M_{0}, 6 M_{0}, 7 M_{0}, 11 M_{0}, 12 M_{0}, 13 M_{0}, 17 M_{0}, 18 M_{0}\right.$, $\left.19 M_{0}\right\}$, which can be grouped into 3 categories: $\left\{d_{1}(m)=\right.$ $\left.6 m M_{0}, 1 \leq m \leq H_{q, p, 1}\right\},\left\{d_{2}(m)=(6(m-1)+1) M_{0}, 1 \leq\right.$ $\left.m \leq H_{q, p, 2}\right\}$, and $\left\{d_{3}(m)=(6 m-1) M_{0}, 1 \leq m \leq H_{q, p, 3}\right\}$, where $H_{q, p, 1}, H_{q, p, 2}$, and $H_{q, p, 3}$ are design parameters. Let $D_{q, p, i}$ denote the total number of periods (including null symbol intervals) of the preamble used to calculate the correlation with the correlation distance $d_{i}$ of the $p$-th part in the $q$ th band, counting from the first non-zero preamble period to the last non-zero preamble period used in the correlation term. $D_{q, p, 1}$ is a special case, which is separated into $D_{q, p, 1}^{o}$ and $D_{q, p, 1}^{e}$, corresponding to the correlation distance $6 m M_{0}$ on the odd symbol indices and the even symbol indices, respectively. Then $N_{q, p, 1}^{o}=D_{q, p, 1}^{o} M_{0}, N_{q, p, 1}^{e}=D_{q, p, 1}^{e} M_{0}$, and $N_{q, p, i}=D_{q, p, i} M_{0}$ for $i=2,3$.

For the correlation term with a correlation distance $d_{i}(m)$, (26) becomes

$$
\theta_{q_{-} p, i}(m) \simeq v_{q}+\frac{N}{2 \pi d_{i}(m)} \frac{\Im\left\{G_{q_{\_} p, i}(m)\right\}+\Im\left\{\mathcal{N}_{q_{\_} p, i}(m)\right\}}{Q_{q_{-} p, i}(m) E_{q_{\_} p}}
$$

where

$$
\begin{aligned}
& Q_{q_{\_} p, 1}(m)=Q_{q_{\_} p, 1}^{o}(m)+Q_{q_{-} p, 1}^{e}(m) \\
& Q_{q_{\_} p, 1}^{o}(m)=\left\lfloor\frac{N_{q_{\_} p, 1}^{o}-d_{1}(m)-M_{0}}{6 M_{0}}\right\rfloor+1 \\
& Q_{q_{\_} p, 1}^{e}(m)=\left\lfloor\frac{N_{q_{\_} p, 1}^{e}-d_{1}(m)-M_{0}}{6 M_{0}}\right\rfloor+1 \\
& Q_{q_{\_} p, i}(m)=\left\lfloor\frac{N_{q_{-} p, i}-d_{i}(m)-M_{0}}{6 M_{0}}\right\rfloor+1 \text { for } i=2,3
\end{aligned}
$$

$G_{q \_p, i}(m)$ and $\mathcal{N}_{q_{\_} p, i}(m)$ are the same as $G_{q \_p}(m)$ and $\mathcal{N}_{q \_p}(m)$, respectively, except that $d(m)$ is replaced by $d_{i}(m)$. Then the covariance matrix $\boldsymbol{C}_{\theta_{q_{-}}}$can be expressed as

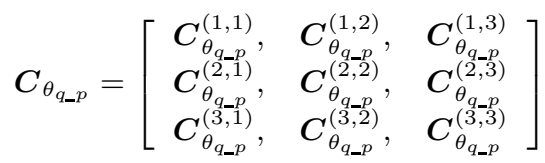

where $\boldsymbol{C}_{\theta_{q_{-} p}}^{(i, l)}$ is the cross-covariance matrix of $\left\{\theta_{q_{\_} p, i}(m)\right\}$ and $\left\{\theta_{q_{\_} p, l}(n)\right\}$, and elements of $\boldsymbol{C}_{\theta_{q_{-} p}}^{(i, l)}$ are given by (34)-(37) as defined below:

$$
\begin{aligned}
& \left.\boldsymbol{C}_{\theta_{q}}^{(1, i)}(m, n)\right|_{i=2,3}=\frac{N^{2} \sigma^{2}}{8 \pi^{2} E_{q}} \frac{1}{d_{1}(m) d_{i}(n) Q_{q, 1}(m) Q_{q, i}(n)} \\
& \int\left(\min \left(Q_{q, 1}^{e}(m), Q_{q, i}(n)\right)\right. \\
& \times\left\{\begin{array}{c}
\left.\quad \min \left(Q_{q, 1}^{o}(m), Q_{q, i}(n)\right)\right), \text { if } d_{1}(m)+d_{i}(n) \geq N_{q, i} \\
\left(\min \left(Q_{q, 1}^{e}(m), Q_{q, i}(n)\right)+\min \left(Q_{q, 1}^{o}(m), Q_{q, i}(n)\right)\right. \\
\left.-2\left(Q_{q, i}(n)-m\right)\right), \quad \text { else. }
\end{array}\right. \\
& \left.\boldsymbol{C}_{\theta_{q}}^{(i, i)}(m, n)\right|_{i=2,3}=\frac{N^{2} \sigma^{2}}{4 \pi^{2} E_{q}} \frac{1}{d_{i}(m) d_{i}(n) Q_{q, i}(m) Q_{q, i}(n)} \\
& \times \begin{cases}Q_{q, i}(m)+\frac{Q_{q, i}(m) N \sigma^{2}}{2 E_{q}}, & \text { if } m=n \\
\min \left(Q_{q, i}(m), Q_{q, i}(n)\right), & \text { else. }\end{cases}
\end{aligned}
$$

Note that $\boldsymbol{C}_{\theta_{q_{\_} p}}$ is Hermitian symmetric.

\section{Method B}

Method B uses another high SNR approximation of (17) as

$$
\theta_{q, p}(m) \simeq v_{q}+\frac{N}{2 \pi d(m)} \frac{\Im\left\{G_{q, p}(m)\right\}}{Q_{q, p}(m) E_{q, p}} .
$$

After straightforward calculation using (38), $\boldsymbol{C}_{\theta_{q, p}}$ can be obtained for the different preamble patterns as follows.

1) Preamble Patterns $1 \& 2$ : The $(m, n)$ th element of $\boldsymbol{C}_{\theta_{q, p}}$ can be expressed as

$$
\begin{array}{r}
\boldsymbol{C}_{\theta_{q}}(m, n)=\frac{N^{2} \sigma^{2}}{4 \pi^{2}\left(3 M_{0}\right)^{2} E_{q}} \frac{1}{m n\left(L_{q}-m\right)\left(L_{q}-n\right)} \\
\quad \times \begin{cases}\min (m, n), & \text { if } m+n<L_{q} \\
L_{q}-\max (m, n), & \text { if } m+n \geq L_{q} .\end{cases}
\end{array}
$$

2) Preamble Patterns $3 \& 4: \boldsymbol{C}_{\theta_{q_{-} p}}$ is given by (33) where $\left\{\boldsymbol{C}_{\theta_{q_{-} p}}^{(i, l)}(m, n)\right\}$ for $i \neq l$ are the same as (37),(35), while those for $i=l$ are given by (40) and (41) as follows:

$$
\left.C_{\theta_{q}}^{(i, i)}(m, n)\right|_{i=2,3}=\frac{N^{2} \sigma^{2}}{4 \pi^{2} E_{q}} \frac{1}{d_{i}(m) d_{i}(n) \max \left(Q_{q, i}(m), Q_{q, i}(n)\right)} .
$$

\section{Abrupt Channel Change Detection And PREAMBLE SEPARATION}

Since the correlation-based estimators require identical channel output preamble symbols, we introduce a simple abrupt channel change detection algorithm, by which we obtain the symbol index where the abrupt channel change occurs. Due to the ambiguity in finding the exact symbol index of the abrupt channel change, we discard two symbols (the detected, $\mathrm{PS}_{i}$, and that which immediately follows, $\left.\mathrm{PS}_{(i+1)}\right)$ in our OFO estimation.

The abrupt channel change results in a variation of channel output symbol energy which we exploit in our detection. For 


$$
\begin{aligned}
& \boldsymbol{C}_{\theta_{q}}^{(2,3)}(m, n)=\frac{N^{2} \sigma^{2}}{4 \pi^{2} E_{q}} \frac{1}{d_{2}(m) d_{3}(n) Q_{q, 2}(m) Q_{q, 3}(n)} \\
& \quad \times \begin{cases}-\frac{1}{2}\left(\left\langle\frac{N_{q, 3}-M_{0}}{6 M_{0}}\right\rangle+\left\langle\frac{N_{q, 2}-M_{0}}{6 M_{0}}\right\rangle\right)+m+n-2, & \text { if } d_{2}(m)+d_{3}(n)<\min \left(N_{q, 2}, N_{q, 3}\right) \\
-\frac{1}{2}\left(\left\langle\frac{\max \left(N_{q, 2}, N_{q, 3}\right)-M_{0}}{6 M_{0}}\right\rangle-m-n+2\right), & \text { if } \min \left(N_{q, 2}, N_{q, 3}\right) \leq d_{2}(m)+d_{3}(n)<\max \left(N_{q, 2}, N_{q, 3}\right) \\
0, & \text { else. }\end{cases} \\
& \boldsymbol{C}_{\theta_{q}}^{(1,1)}(m, n)=\frac{N^{2} \sigma^{2}}{4 \pi^{2} E_{q}} \frac{1}{d_{1}(m) d_{1}(n) Q_{q, 1}(m) Q_{q, 1}(n)} \\
& \times \begin{cases}2 \min (m, n), \\
\min (m, n)+\min \left(Q_{q, 1}^{o}(m), Q_{q, 1}^{e}(m), Q_{q, 1}^{o}(n), Q_{q, 1}^{e}(n)\right), & \text { if } \min \left(N_{q, 1}^{o}, N_{q, 1}^{e}\right) \leq d_{1}(m)+d_{1}(n)<\max \left(N_{q, 1}^{o}, N_{q, 1}^{e}\right) \\
\min \left(Q_{q, 1}^{o}(m), Q_{q, 1}^{o}(n)\right)+\min \left(Q_{q, 1}^{e}(m), Q_{q, 1}^{e}(n)\right), & \text { if } d_{1}(m)+d_{1}(n) \geq \max \left(N_{q, 1}^{o}, N_{q, 1}^{e}\right)\end{cases}
\end{aligned}
$$

\begin{tabular}{|c|c|c|c|}
\hline \multicolumn{4}{|c|}{ Preamble Pattern 1 (or 2) } \\
\hline & conditions of $\left\{\hat{a}_{1}, \hat{a}_{2}, \hat{a}_{3}\right\}$ & $L_{q}^{(1)}$ & $L_{q}^{(2)}$ \\
\hline & if $\hat{a}_{1}=\hat{a}_{2}=\hat{a}_{3}$ & $\hat{a}_{1}, \hat{a}_{2}, \hat{a}_{3}-1$ & $L_{1}-\hat{a}_{1}-1, L_{2}-\hat{a}_{2}, L_{3}-\hat{a}_{3}$ \\
\hline & else if $\hat{a}_{1}=\hat{a}_{2}=\hat{a}_{3}+1$ & $\hat{a}_{1}, \hat{a}_{2}-1, \hat{a}_{3}$ & $L_{1}-\hat{a}_{1}, L_{2}-\hat{a}_{2}, L_{3}-\hat{a}_{3}-1$ \\
\hline & else if $\hat{a}_{1}-1=\hat{a}_{2}=\hat{a}_{3}$ & $\hat{a}_{1}-1, \hat{a}_{2}, \hat{a}_{3}$ & $L_{1}-\hat{a}_{1}, L_{2}-\hat{a}_{2}-1, L_{3}-\hat{a}_{3}$ \\
\hline$\dagger$ & else if $\hat{a}_{1}=\hat{a}_{2}=1$ & $1,0,0$ & $L_{1}-1, L_{2}-1, L_{3}-1$ \\
\hline$\dagger$ & else if $\hat{a}_{1}=1$ & $0,0,0$ & $L_{1}-1, L_{2}-1, L_{3}$ \\
\hline$\dagger$ & else if $\hat{a}_{2}=L_{2}-1 \& \hat{a}_{3}=L_{3}-1$ & $L_{1}-1, L_{2}-1, L_{3}-1$ & $0,0,1$ \\
\hline$\dagger$ & else if $\hat{a}_{3}=L_{3}-1$ & $L_{1}, L_{2}-1, L_{3}-1$ & $0,0,0$ \\
\hline$\star$ & else & $L_{1}, L_{2}, L_{3}$ & $0,0,0$ \\
\hline \multicolumn{4}{|c|}{ Preamble Pattern 3 (or 4) } \\
\hline & $\{2,2,2\},\{4,4,4\}$ & $\hat{a}_{1}, \hat{a}_{2}, \hat{a}_{3}-1$ & $L_{1}-\hat{a}_{1}-1, L_{2}-\hat{a}_{2}, L_{3}-\hat{a}_{3}$ \\
\hline & $\{2,2,1\},\{4,4,3\},\{6,6,5\}$ & $\hat{a}_{1}, \hat{a}_{2}, \hat{a}_{3}-1$ & $L_{1}-\hat{a}_{1}, L_{2}-\hat{a}_{2}, L_{3}-\hat{a}_{3}-1$ \\
\hline & $\{4,4,2\},\{6,6,4\}$ & $\hat{a}_{1}, \hat{a}_{2}-1, \hat{a}_{3}$ & $L_{1}-\hat{a}_{1}, L_{2}-\hat{a}_{2}, L_{3}-\hat{a}_{3}-1$ \\
\hline & $\{4,3,2\},\{6,5,4\}$ & $\hat{a}_{1}, \hat{a}_{2}-1, \hat{a}_{3}$ & $L_{1}-\hat{a}_{1}, L_{2}-\hat{a}_{2}-1, L_{3}-\hat{a}_{3}$ \\
\hline & $\{4,2,2\},\{6,4,4\}$ & $\hat{a}_{1}-1, \hat{a}_{2}, \hat{a}_{3}$ & $L_{1}-\hat{a}_{1}, L_{2}-\hat{a}_{2}-1, L_{3}-\hat{a}_{3}$ \\
\hline & $\{3,2,2\},\{5,4,4\}$ & $\hat{a}_{1}, \hat{a}_{2}-1, \hat{a}_{3}$ & $L_{1}-\hat{a}_{1}-1, L_{2}-\hat{a}_{2}, L_{3}-\hat{a}_{3}$ \\
\hline$+\dagger$ & else if $\hat{a}_{1}=2 \& \hat{a}_{2}=1$ & $2,0,0$ & $L_{1}-2, L_{2}-2, L_{3}$ \\
\hline $\bar{\dagger}$ & else if $\hat{a}_{1}=2 \& \hat{a}_{2}=2$ & $2,0,0$ & $L_{1}-2, L_{2}-2, L_{3}-1$ \\
\hline$\dagger$ & else if $\hat{a}_{1}=1$ & $0,0,0$ & $L_{1}-2, L_{2}, L_{3}$ \\
\hline$\dagger$ & else if $\hat{a}_{1}=L_{1}-1 \& \hat{a}_{2}=L_{2}-1$ & $L_{1}-2, L_{2}-1, L_{3}$ & $0,1,0$ \\
\hline$\dagger$ & else if $\hat{a}_{1}=L_{1}-2 \& \hat{a}_{2}=L_{2}-1$ & $L_{1}-2, L_{2}-1, L_{3}-1$ & $1,1,0$ \\
\hline$+\dagger$ & else if $\hat{a}_{2}=L_{2}-1$ & $L_{1}-1, L_{2}-1, L_{3}$ & $0,0,0$ \\
\hline$\frac{1}{\star}$ & else & $L_{1}, L_{2}, L_{3}$ & $0,0,0$ \\
\hline
\end{tabular}

TABLE I

MAPPING SCHEME IN THE ABRUPT CHANNEL CHANGE DETECTION AND PREAMBLE SEPARATION

each band, the symbol index corresponding to the abrupt channel change is estimated as

$$
\widehat{a}_{q}=\underset{1 \leq a_{q} \leq L_{q}-1}{\arg \max }\left|\frac{\sum_{l=1}^{a_{q}} \widehat{E}_{l, q}}{a_{q}}-\frac{\sum_{l=a_{q}+1}^{L_{q}} \widehat{E}_{l, q}}{L_{q}-a_{q}}\right|
$$

where $\widehat{E}_{l, q}=\sum_{i=0}^{N-1}\left|r_{q}\left(t_{l}^{q}(i)\right)\right|^{2}, \quad q=1,2,3$.

Every channel change will give a corresponding pattern $\left(\widehat{a_{1}}\right.$, $\left.\widehat{a_{2}}, \widehat{a_{3}}\right)$. If there is no change for the preamble in a particular $q$-th band, then $\widehat{a_{q}}$ would be uniformly distributed in the range $\left[1, L_{q}-1\right]$ due to the random noise effect. After obtaining $\left\{\widehat{a_{q}}\right\}$, we separate the preamble in each band into two parts (left and right). The first contains the first $L_{q}^{(1)}$ non-zero preamble symbols and the second contains the last $L_{q}^{(2)}$ nonzero preamble symbols of the $q$-th band, where the superscript

(1) and (2) denote the left and right parts, respectively.

We tabulate the mapping from $\left\{\widehat{a_{q}}\right\}$ to $L_{q}^{(1)}$ and $L_{q}^{(2)}$ in Table I for all preamble patterns. If $\left(\widehat{a_{1}}, \widehat{a_{2}}, \widehat{a_{3}}\right)$ corresponds to any of the rows except the ones marked with $\star$ in the tables, the preambles can be separated into the first $L_{q}^{(1)}$ and the last $L_{q}^{(2)}$ symbols; otherwise we suppose no abrupt channel change occurs during the preamble transmission. Note that the mapping algorithm is sequentially performed down the tables. The rows marked with $\dagger$ correspond to the situations when the channel changes abruptly during the first or last few symbols of the whole 21 symbols, hence change can be observed in only one or two of the three bands.

\section{DESIGN PARAMETERS AND DisCUSSIONS}

We prove in Appendix A that $\boldsymbol{C}_{\theta_{q}}$ gives the minimum BLUE variance in (45) for the maximum size full-rank matrix $\boldsymbol{C}_{\theta_{q}}$. This fact yields the best design parameters for the considered system as follows. For Method A, we obtain $H_{q}=L_{q}-1$ for the preamble patterns 1 and 2 , and $H_{q, 1}=\left\lfloor\left(\max \left(D_{q, 1}^{o}, D_{q, 1}^{e}\right)-1\right) / 6\right\rfloor, H_{q, 2}=\left\lfloor\left(D_{q, 2}+5\right) / 6\right\rfloor$, and $H_{q, 3}=\left\lfloor D_{q, 3} / 6\right\rfloor$ for the patterns 3 and 4 , (i.e., $H_{q}=6$ for the pattern 1 or $2, H_{1,2}=4, H_{1,1}=H_{1,3}=H_{2,2}=$ $H_{2,3}=H_{3,2}=3, H_{2,1}=H_{3,1}=H_{3,3}=2$ for the pattern 3 or 4). For method $\mathrm{B}$, we have $H_{q}=\left\lfloor L_{q} / 2\right\rfloor$ for the patterns 1 and 2 , and $H_{q, 1}=\left\lfloor\left(\max \left(D_{q, 1}^{o}, D_{q, 1}^{e}\right)+5\right) / 12\right\rfloor, H_{q, 2}=$ $\left\lfloor\left(D_{q, 2}+10\right) / 12\right\rfloor, H_{q, 3}=\left\lfloor\left(D_{q, 3}+6\right) / 12\right\rfloor$ for the patterns 3 and 4 . The design parameters for the preamble pattern 1 or 2 are much simpler than those for pattern 3 or 4 due to its periodic preamble structure. The above parameter design can 
TABLE II

Computational Complexities of The Proposed Methods

\begin{tabular}{|c|c|c|}
\hline & Preamble Pattern 1 or 2 & Preamble Pattern 3 or 4 \\
\hline \multirow{2}{*}{ \# ERM } & $\sum_{p=1}^{2} \sum_{q=1}^{3}\left\{4 N L_{q_{\_} p} H_{q_{\_} p}\right.$ & $4 N \cdot \sum_{p=1}^{2} \sum_{q=1}^{3} \sum_{i=1}^{3} \sum_{m=1}^{H_{q_{\_} p, i}}\left\{Q_{q_{\_} p, i}(m)\right\}$ \\
& $\left.-2 N H_{q_{\_} p}\left(H_{q_{\_} p}+1\right)+H_{q_{\_} p}\right\}$ & $+\sum_{p=1}^{2} \sum_{q=1}^{3} \sum_{i=1}^{3}\left\{H_{q_{\_} p, i}\right\}$ \\
\hline \multirow{2}{*}{ \# ERA } & $\sum_{p=1}^{2} \sum_{q=1}^{3}\left\{4 N L_{q_{\_} p} H_{q_{\_} p}\right.$ & $4 N \cdot \sum_{p=1}^{2} \sum_{q=1}^{3} \sum_{i=1}^{3} \sum_{m=1}^{H_{q_{\_} p, i}}\left\{Q_{q_{\_} p, i}(m)\right\}$ \\
& $\left.-2 N H_{q_{\_} p}\left(H_{q_{\_} p}+1\right)-H_{q_{\_} p}\right\}-1$ & $-\sum_{p=1}^{2} \sum_{q=1}^{3} \sum_{i=1}^{3}\left\{H_{q_{\_} p, i}\right\}-1$ \\
\hline \# angle $\{\}$. & $\sum_{p=1}^{2} \sum_{q=1}^{3}\left\{H_{q_{\_} p}\right\}$ & $\sum_{p=1}^{2} \sum_{q=1}^{3} \sum_{i=1}^{3}\left\{H_{q_{\_} p, i}\right\}$ \\
\hline
\end{tabular}

also be applied to other multi-band systems by appropriately changing the relationship between $\left\{H_{q, i}\right\}$ and $\left\{D_{q, i}\right\}$.

For method A, the weighting values for each band depend on $E_{q, p} /\left(N \sigma^{2}\right)$ while for method B they do not. However, for method $\mathrm{A}$ with the best design parameters mentioned above, the weighting values for each band are insensitive to the $E_{q, p} /\left(N \sigma^{2}\right)$ values of practical interest (see Fig. 3 in [36]). Hence, a fixed design value (say 10) for $E_{q, p} /\left(N \sigma^{2}\right)$ can be used when the weighting values are pre-computed.

Next, we observe that the covariance matrices $\left\{\boldsymbol{C}_{\theta_{q, p}}\right\}$ are inversely proportional to $E_{q, p}$, and the estimator in (20) or (23) requires the knowledge of $E_{q, p}$. Here, we replace $E_{q, p}$ with its estimate $\widehat{E}_{q, p}$ which can be obtained by simply averaging the non-zero received symbol energies of the $p$-th preamble part in the $q$-th band as

$$
\widehat{E}_{q, p}=\sum_{l=1}^{L_{q, p}} \sum_{k=0}^{N-1}\left|r_{q}\left(t_{l}^{q, p}(k)\right)\right|^{2} / L_{q, p} .
$$

The variance for the BLUE method using the $p$-th preamble part in the $q$-th band is

$$
\operatorname{Var}\left\{\widehat{v}_{q, p}\right\}=\left(\mathbf{1}^{T} \boldsymbol{C}_{\theta_{q, p}}^{-1} \mathbf{1}\right)^{-1} \triangleq \frac{1}{A_{q, p} E_{q, p}} .
$$

Hence, our proposed estimator in (23) can be expressed as

$$
\widehat{v}=\frac{\sum_{p=1}^{2} \sum_{q=1}^{3} E_{q, p} A_{q, p} b_{q} \widehat{v}_{q, p}}{\sum_{p=1}^{2} \sum_{q=1}^{3} \widehat{E}_{q, p} A_{q, p} b_{q}^{2}} .
$$

Note that the weighting values of the estimator depend on the position of the abrupt channel change. We can pre-compute the weighting value sets for all possible abrupt channel change positions and store them at the receiver for easy use in the estimation.

In (16), we require $\left|v_{q}\right|<N /(2 d(m))$ to avoid estimation ambiguity. For the correlation terms with large correlation distances $\{d(m)\}$, this condition limits the estimation range. This issue can be easily circumvented by performing initial frequency offset compensation on the received preamble signal, based on the initial frequency offset estimate obtained from the correlation term(s) with small correlation distance(s). For the preamble pattern 1 or 2 , using $\left\{R_{q}(d(1))\right\}$ for the initial frequency offset compensation will extend the estimation range to $\left.\left|\widehat{v}_{q}\right|<N /(2 d(1)) \simeq 0.129\right)$, which is more than sufficient, since the maximum possible carrier frequency offset for the $20 \mathrm{ppm}$ oscillator accuracy specified in [8] is just $\left|v_{q}\right|_{\text {max }}=0.04096 b_{q}$ (see (7) for $b_{q}$ ).

In practice, sync detection and timing synchronization are performed before the CFO estimation. For simpler practical implementation, the first few preamble symbols are typically used for sync detection and (coarse) timing synchronization, and the remaining preamble symbols are used for CFO estimation. In alternative implementation, $\mathrm{CFO}$ is estimated based on all 21 preamble symbols by using an appropriate buffer size. In this paper, we use all 21 preamble symbols in the CFO estimation. However, our method can also be applied to the former implementation by changing the values of the parameters $L_{q}, D_{q, p, i}, N_{q, p}$, and $H_{q, p}$, accordingly.

The computational complexities of the proposed methods are relatively low since the methods are based on correlation. The exact complexities in terms of the numbers of equivalent real multiplication (ERM), equivalent real addition (ERA), and the angle operation are presented in Table II for the proposed method, except for the computation of $\widehat{E}_{q, p}$, which can be obtained from the timing synchronization or automatic gain control stage. As a numerical example for complexity, if we use the design parameters mentioned at the beginning of this section for method A, the numbers of angle operation, ERM, and ERA are respectively 18, 32274, and 32237 for the preamble patterns 1 and 2, and 26, 32794, and 32741 for the preamble patterns 3 and 4 .

\section{Simulation Results and Discussions}

We use the simulation parameters as specified in [8]: $N=128, N_{g}=37$, carrier frequencies $f_{1}=3432 \mathrm{MHz}$, $f_{2}=3960 \mathrm{MHz}, f_{3}=4488 \mathrm{MHz}$, the sub-carrier spacing $1 / T=4.125 \mathrm{MHz}$, and four different preambles. The channel model CM-2 with $L_{h}=6$ and $K=6$ is adopted. We exclude the lognormal shadowing factor $X$ and hence the realizations of lognormal components in the channel gains $\left\{\alpha_{l, k}\right\}$ are not normalized. $p(t)$ is a spectral raised cosine pulse with a filter span of $\left[-5 T_{s}, 5 T_{s}\right]$. The normalized OFO $v$ is set to 0.01 . The non-zero transmitted preamble samples are normalized to obtain a unit average sample energy. For the time-variant UWB channel, we assume the channel transition probability matrix is $\boldsymbol{P}=[0.9,0.1 ; 0.6,0.4]$ and the steadystate probability vector is $[0.8,0.2]^{T}$. We use $\mathcal{J}=K$ and $k_{0}=0$ unless mentioned otherwise.

For comparison, we also evaluate two sets of reference estimators. The estimators in the first set are denoted by "Cor. $\left(i_{1}, i_{2}, \ldots, i_{k}\right)$ " in the figures and defined by

$$
\widehat{v}=\frac{1}{3 k} \sum_{q=1}^{3} \sum_{l=1}^{k} \theta_{q}\left(i_{l}\right) / b_{q}
$$

which is simply an average of the estimates $\theta_{q}\left(i_{1}\right), \theta_{q}\left(i_{2}\right)$ ,$\ldots$, and $\theta_{q}\left(i_{k}\right)$ obtained from the correlation terms with correlation distances of $i_{1} M_{0}, i_{2} M_{0}, \ldots$, and $i_{k} M_{0}$, respectively. Note that in the above estimators, we have averaged the estimates across the three bands and have already incorporated the effects of different CFOs in distinct frequency bands. In the second set, the proposed BLUE method is applied to each individual band separately, i.e., the CFO estimation and 


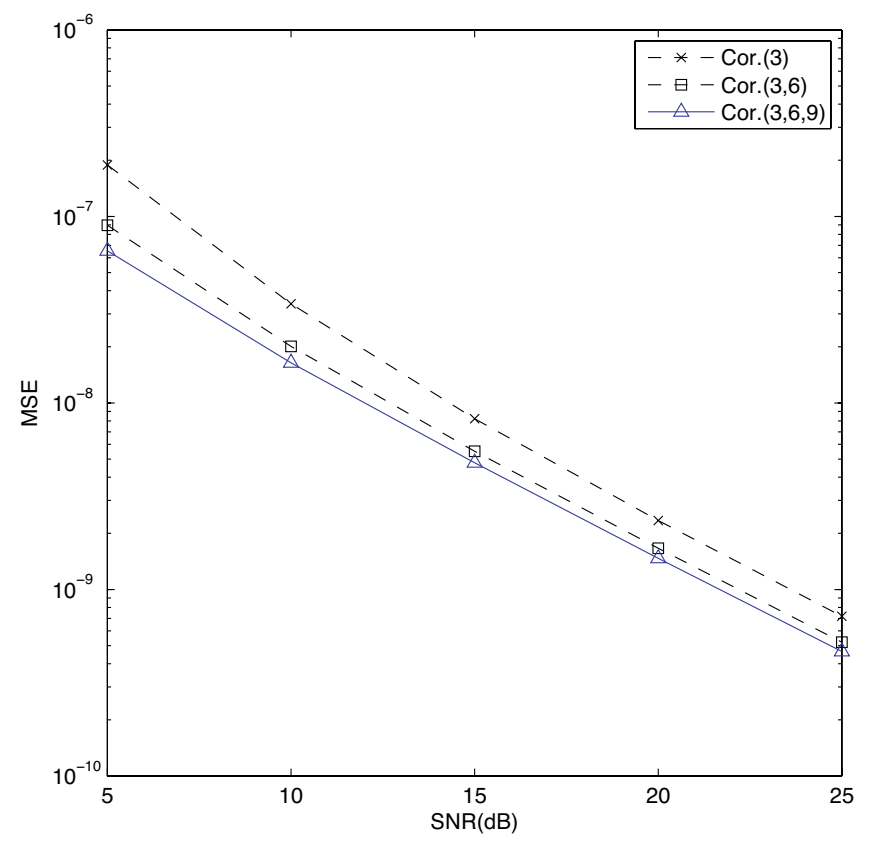

Fig. 3. The normalized OFO estimation performance of the first set of reference methods with different numbers of correlation distances over a timeinvariant channel for preamble pattern 1 (or equivalently 2 ).

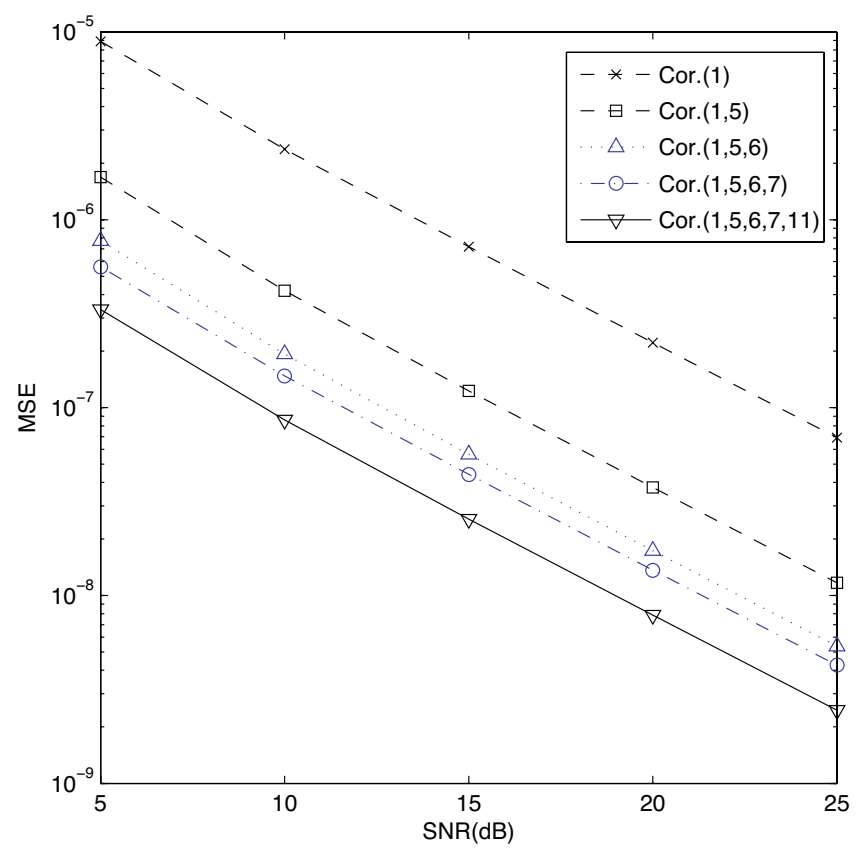

Fig. 4. The normalized OFO estimation performance of the first set of reference methods with different numbers of correlation distances over a timeinvariant channel for preamble pattern 3 (or equivalently 4).

compensation are performed independently in each band and there is no averaging across the three bands.

\section{A. Performance in Time-Invariant UWB Channels}

In Figs. 3 and 4, we present the MSEs of the first set of reference estimators with different numbers of correlation distances for the preamble patterns 1 (or equivalently 2 ) and 3 (or equivalently 4), respectively, for a time-invariant channel. Using more correlation terms improves the MSE performance

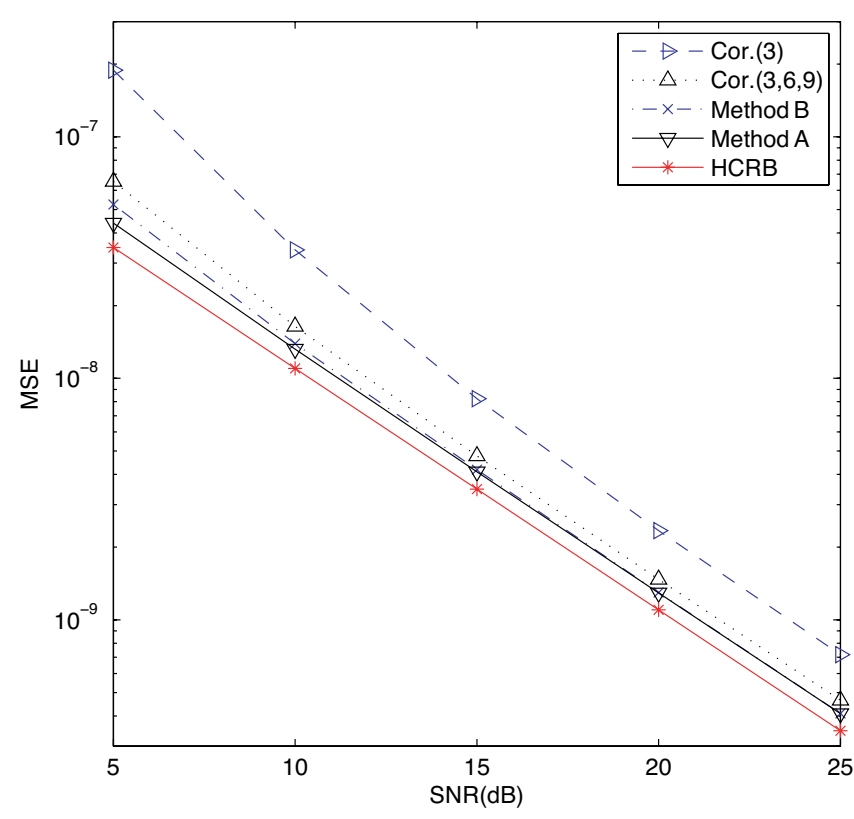

Fig. 5. The normalized OFO estimation performance comparison in a timeinvariant channel for the preamble pattern 1 (or equivalently 2).

at the expense of complexity. Note that if the effects of different CFOs in the three bands were not incorporated in the reference estimators, the performance degradation would be quite large.

Figs. 5 and 6 present the MSEs of the proposed methods (Method $A$ and Method $B$ ) for the preamble patterns 1 (or equivalently 2) and 3 (or equivalently 4), respectively, for a time-invariant channel. Method $A$ gives the minimum MSE, which is quite close to the hybrid Cramer-Rao bound (HCRB) (derived in Appendix B) at moderate and high SNRs. All proposed methods perform better than the first set of reference methods, and the improvements of the proposed methods are more significant for the preamble patterns 3 and 4 .

Next, we compare our proposed method and the second set of reference estimators (the independent BLUE CFO estimation for each band) in Fig. 7. For the convenience of comparison, we convert all CFO estimates to OFO estimates and present the MSE of the OFO estimate. Our proposed OFO estimation method achieves a 4-6.4 dB MSE improvement (a 4-7 dB SNR advantage) over the second set of reference estimators since our estimator has incorporated the frequency diversity of the three bands.

\section{B. Performance in Time-Variant UWB Channels}

If the received preamble energy used in the sync detection is too low, the signal will not be detected and OFO estimation is not needed. This situation is embedded in our evaluation for time-variant channels as follows. The OFO estimation is skipped if

(i) $\sum_{q=1}^{3} \sum_{l=1}^{L_{q}}\left\{\widehat{E}_{l, q} /\left(3 L_{q} N \sigma^{2}\right)\right\}<\gamma$ when there is no abrupt channel change,

(ii) $\sum_{q=1}^{3}\left\{\widehat{E}_{q, p}\right\} /\left(3 N \sigma^{2}\right)<\gamma$ for all $p$ when there is an abrupt channel change,

where $\gamma$ is a threshold value. 


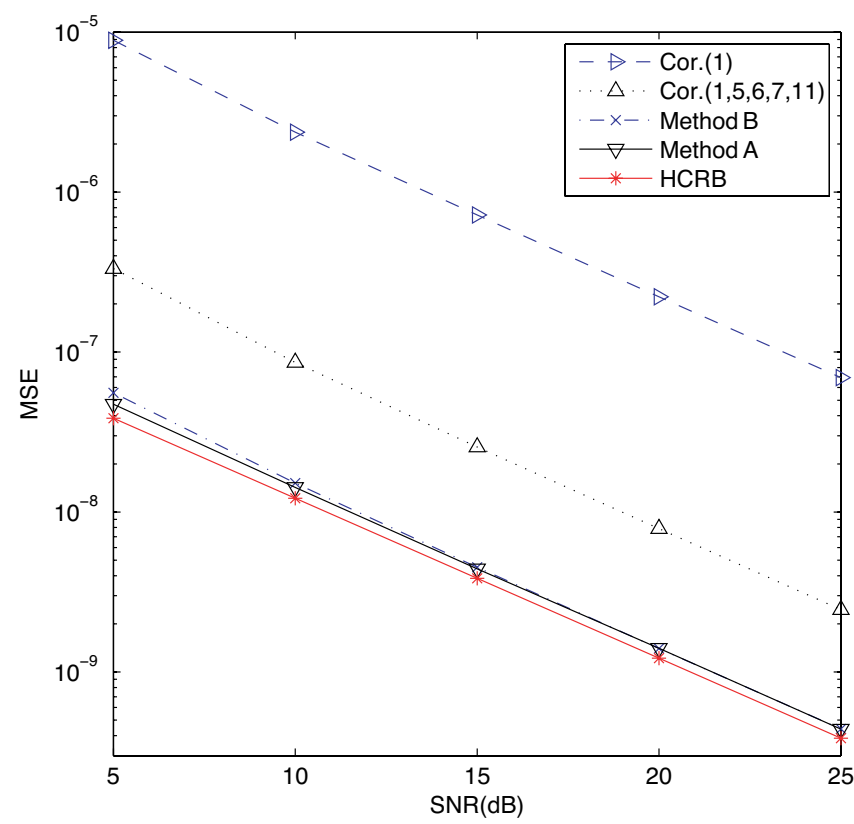

Fig. 6. The normalized OFO estimation performance comparison between the proposed method and the first set of reference methods in a time-invariant channel for the preamble pattern 3 (or equivalently 4 ).

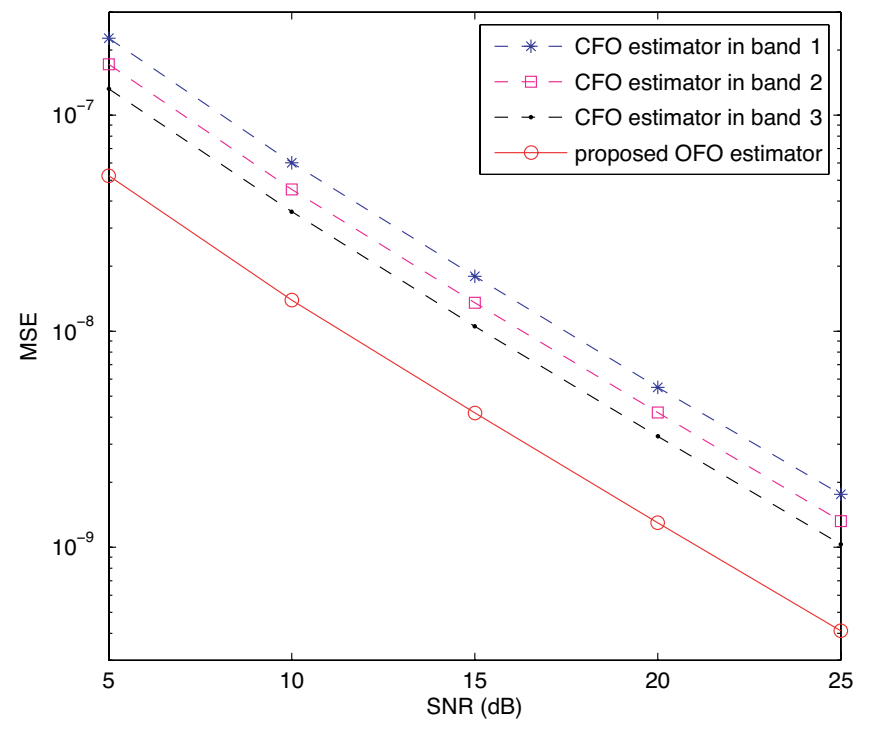

Fig. 7. The normalized OFO estimation performance comparison between the proposed method and the second set of reference methods (independent CFO estimation on each band) in a time-invariant UWB channel for the preamble pattern 1 (or equivalently 2 ).

If the abrupt channel change results in an increase of the received snap-shot SNR from below to above the SNR threshold of the sync detection (from bad to good SNR), the preamble part before the abrupt channel change would not be detected and hence, is not used in the OFO estimation. In addition, if the number of preamble symbols with good SNR in each band is less than a certain value $\zeta$ (we use $\zeta=3$ for the pattern 1 (or 2) and $\zeta=4$ for the pattern 3 (or 4 ) in our simulation), neither the OFO estimation nor data detection would be reliable ${ }^{6}$. Hence, we skip the OFO estimation (and

${ }^{6}$ Recall that the operating SNR of UWB systems is low.

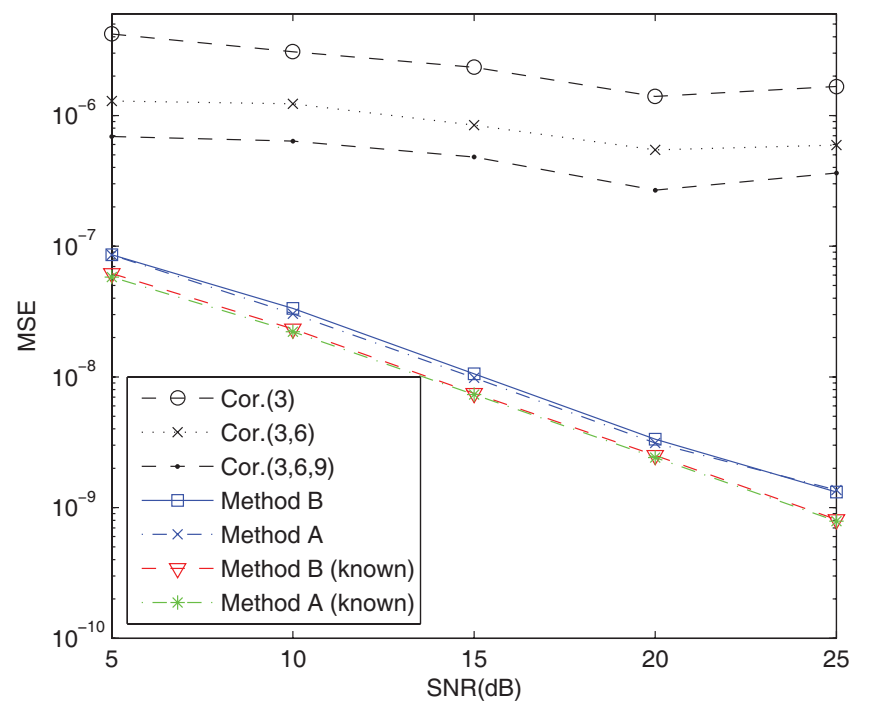

Fig. 8. The normalized OFO estimation performance comparison in a time-variant UWB channel for the preamble pattern 1 (or equivalently 2). (The proposed methods with perfect knowledge of the abrupt channel change instant are denoted by "known.")

data detection) for this case.

If the abrupt channel change results in a drop of the received snap-shot SNR below the SNR threshold, the receiver would not be able to detect the remaining data correctly. Hence, for the sake of energy saving, the whole packet should be discarded without processing anything. We address this issue by skipping the OFO estimation if $\sum_{q=1}^{3}\left\{\widehat{E}_{q, p}\right\} /\left(3 N \sigma^{2}\right)<\gamma$. In our simulation, we use $\gamma=4 \mathrm{~dB}$ for all OFO estimators.

The MSE comparisons of the proposed OFO estimators and the first set of reference estimators over a time-variant UWB channel are presented in Figs. 8 and 9 for the preamble patterns 1 (or 2) and 3 (or 4), respectively. The degradation of the MSE performance of the proposed methods in a timevariant channel compared with the proposed methods in a time-invariant channel is due to: (1) the channel energy loss, (2) smaller correlation distances, and (3) the occasional errors of the abrupt channel change detection algorithm. Both proposed methods perform better than the reference estimators. Method $A$ performs marginally better than Method $B$ at lower SNR while the two methods perform almost the same at higher SNR. The proposed methods with the abrupt channel change detection experience slight MSE degradation if compared to the proposed methods with perfect knowledge of the abrupt channel change instant. However, our proposed methods give significant (5 to $20 \mathrm{~dB}$ ) MSE advantages over the reference estimators.

In Fig. 10, we evaluate the proposed method in two timevariant channels. In the first channel, we use $\mathcal{J}=K$ and $k_{0}=$ 0 , i.e., the whole cluster is blocked when a moving scatterer affects the channel. In the second channel, we use $\mathcal{J}=2$, i.e., only two adjacent rays are blocked when a moving scatterer affects the channel. We only apply method B here since the estimation performance of the other method is similar. The simulation result shows that the proposed method works well in both channels. The performance is slightly better in the second channel than in the first channel. This result is expected 


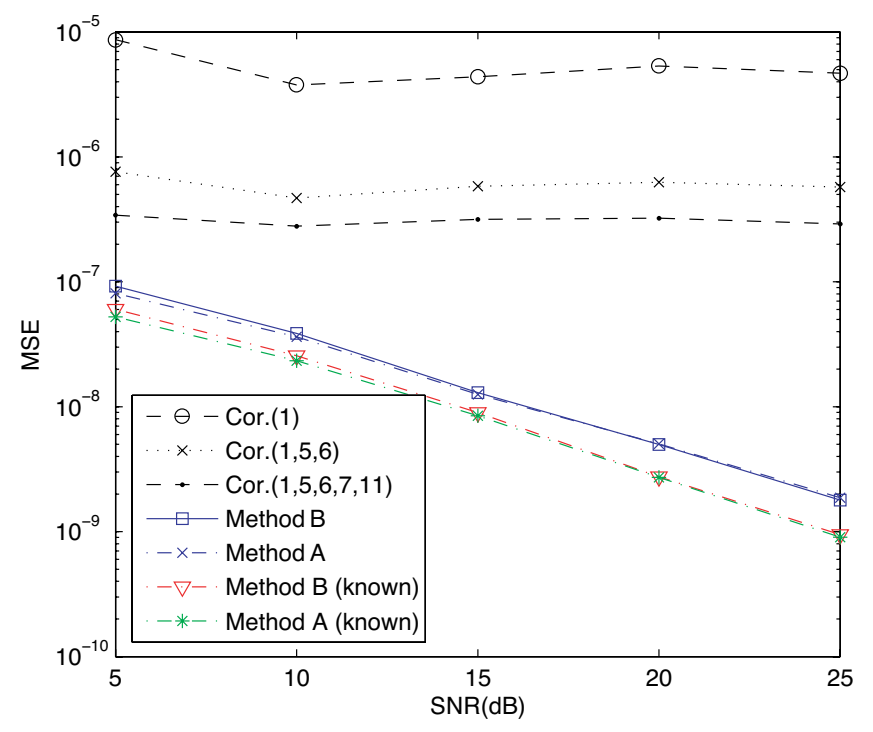

Fig. 9. The normalized OFO estimation performance comparison in a time-variant UWB channel for the preamble pattern 3 (or equivalently 4). (The proposed methods with perfect knowledge of the abrupt channel change instant are denoted by "known.")

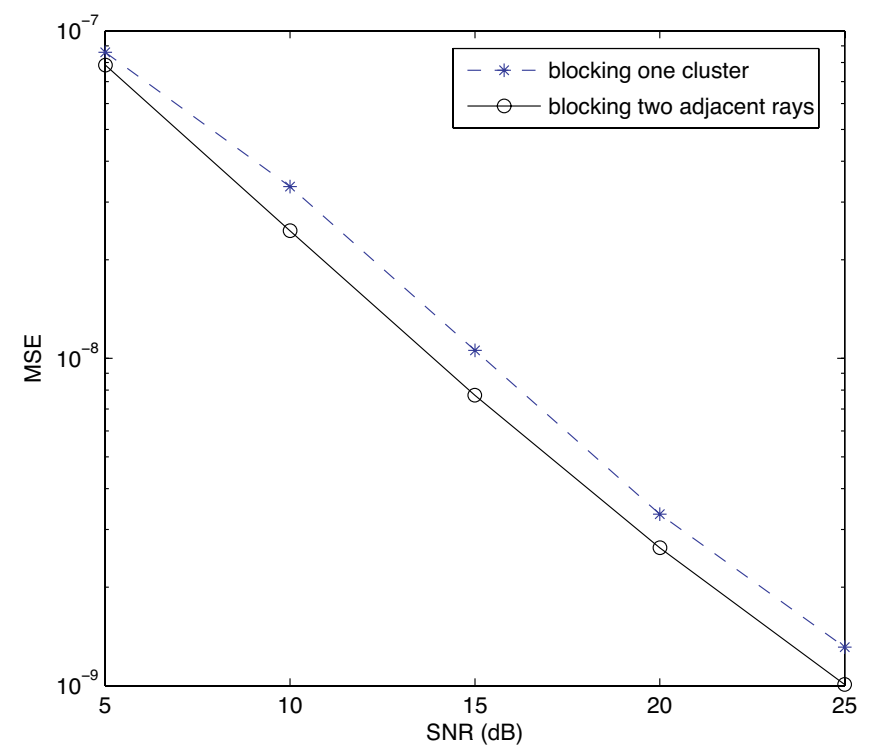

Fig. 10. The normalized OFO estimation performance of the proposed method in two time-variant channels (defined by blocking one whole cluster and blocking two adjacent rays, respectively, when an abrupt channel change occurs) for the preamble pattern 1 (or equivalently 2 ).

since the effect of the moving scatterer on the channel is more severe in the first channel.

\section{Conclusions}

We have presented enhanced OFO estimators based on the BLUE principle for MB-OFDM based UWB systems by exploiting several characteristics of the MB-OFDM system such as different CFOs, channel responses, received energies and preamble structures in different bands. We develop our estimators to be robust against sudden channel changes which can occur in some UWB systems with fast moving scatters. The proposed estimators are adaptive such that when a sudden channel change is detected during the preamble duration, the estimator designed for such a time-variant scenario is used, otherwise the estimator developed for the time-invariant channel is applied. For systems with no sudden channel changes, the latter estimator can be solely implemented for implementation simplicity. Our proposed approach can be applied to other multi-band systems or similar frequencyhopped systems.

\section{APPENDIX A}

The parameter $\left\{H_{q}\right\}$ of the proposed estimators can be optimized by minimizing the BLUE variance. In this Appendix, we will prove that the maximum size full-rank covariance matrix gives the minimum BLUE variance, which in turn yields the design values of $\left\{H_{q}\right\}$ as mentioned in Section V. Consider a full rank covariance matrix $C$ of size $K \times K$. Define

$$
\boldsymbol{C}=\left[\begin{array}{ll}
\boldsymbol{C}_{11} & \boldsymbol{C}_{12} \\
\boldsymbol{C}_{21} & \boldsymbol{C}_{22}
\end{array}\right], \boldsymbol{A}=\boldsymbol{C}^{-1}=\left[\begin{array}{ll}
\boldsymbol{A}_{11} & \boldsymbol{A}_{12} \\
\boldsymbol{A}_{21} & \boldsymbol{A}_{22}
\end{array}\right]
$$

where $\boldsymbol{C}_{11}$ and $\boldsymbol{A}_{11}$ are matrices of size $(K-1) \times(K-1)$, $\boldsymbol{C}_{12}$ and $\boldsymbol{A}_{12}$ are of size $(K-1) \times 1, \boldsymbol{C}_{21}$ and $\boldsymbol{A}_{21}$ are of size $1 \times(K-1)$, and $\boldsymbol{C}_{22}$ and $\boldsymbol{A}_{22}$ are of size $1 \times 1$. Then we have [41]

$$
\begin{aligned}
\boldsymbol{C}^{-1} & =\left[\begin{array}{cc}
\boldsymbol{C}_{11}^{-1}+\boldsymbol{C}_{11}^{-1} \boldsymbol{C}_{12} \boldsymbol{A}_{22} \boldsymbol{C}_{21} \boldsymbol{C}_{11}^{-1} & -\boldsymbol{C}_{11}^{-1} \boldsymbol{C}_{12} \boldsymbol{A}_{22} \\
-\boldsymbol{C}_{22}^{-1} \boldsymbol{C}_{21} \boldsymbol{A}_{11} & \boldsymbol{A}_{22}
\end{array}\right], \\
\mathbf{1}_{K}^{T} \boldsymbol{C}^{-1} \mathbf{1}_{K} & =\mathbf{1}_{K-1}^{T} \boldsymbol{C}_{11}^{-1} \mathbf{1}_{K-1}+\mathbf{1}_{K-1}^{T} \boldsymbol{C}_{11}^{-1} \boldsymbol{C}_{12} \boldsymbol{A}_{22} \boldsymbol{C}_{21} \boldsymbol{C}_{11}^{-1} \mathbf{1}_{K-1} \\
& -\boldsymbol{C}_{22}^{-1} \boldsymbol{C}_{21} \boldsymbol{A}_{11} \mathbf{1}_{k-1}-\mathbf{1}_{K-1}^{T} \boldsymbol{C}_{11}^{-1} \boldsymbol{C}_{12} \boldsymbol{A}_{22}+\boldsymbol{A}_{22}
\end{aligned}
$$

where $\mathbf{1}_{K}$ is the all-one column vector of length $K$.

Due to the Hermitian property of $C$, we can show that

$$
\mathbf{1}_{K}^{T} \boldsymbol{C}^{-1} \mathbf{1}_{K}=\mathbf{1}_{K-1}^{T} \boldsymbol{C}^{-1} \mathbf{1}_{K-1}+\boldsymbol{A}_{\mathbf{2 2}}\left|\mathbf{1}_{K-1}^{T} \boldsymbol{C}_{11}^{-1} \boldsymbol{C}_{12}-1\right|^{2} .
$$

Since $\boldsymbol{C}$ is positive definite, $\boldsymbol{A}$ is positive definite, $\boldsymbol{A}_{22}>0$ and hence, we have

$$
\mathbf{1}_{K}^{T} \boldsymbol{C}^{-1} \mathbf{1}_{K} \geq \mathbf{1}_{K-1}^{T} \boldsymbol{C}^{-1} \mathbf{1}_{K-1} .
$$

The above results along with the BLUE variance equation in (45) prove that the maximum size full-rank covariance matrix gives the minimum BLUE variance.

\section{APPENDIX B}

In this Appendix, we derive the hybrid CRB (HCRB) [40] for OFO estimator in the UWB MB-OFDM system. The received signal in (8) can be expressed in matrix form as

$$
\begin{aligned}
\boldsymbol{r} & =e^{j \varphi} \boldsymbol{W}(v) \boldsymbol{S h}+\boldsymbol{n} \\
\text { where } & =\left[\boldsymbol{r}_{\mathbf{1}}{ }^{T} \boldsymbol{r}_{\mathbf{2}}^{T} \boldsymbol{r}_{\mathbf{3}}^{T}\right]^{T}, \\
\boldsymbol{r}_{q} & =\left[r_{q}(0), r_{q}(1), \ldots, r_{q}(N-1)\right]^{T} \\
\boldsymbol{h} & =\left[\boldsymbol{h}_{\mathbf{1}}^{T} \boldsymbol{h}_{\mathbf{2}}^{T} \boldsymbol{h}_{\mathbf{3}}^{T}\right]^{T}, \\
\boldsymbol{h}_{q} & =\left[h_{q}(0), h_{q}(1), \ldots, h_{q}\left(K^{\prime}\right)\right]^{T} \\
\boldsymbol{W}(v) & =\operatorname{diag}\left\{\boldsymbol{W}_{\mathbf{1}}(v), \boldsymbol{W}_{\mathbf{2}}(v), \boldsymbol{W}_{\mathbf{3}}(v)\right\} \\
\boldsymbol{W}_{\boldsymbol{q}}(v) & =\operatorname{diag}\left\{1, e^{j 2 \pi b_{q} v / N}, e^{j 2 \pi 2 b_{q} v / N}, \ldots,\right. \\
\left.e^{j 2 \pi(N-1) b_{q} v / N}\right\} & \\
\boldsymbol{n} & =[n(0), n(1), \ldots, n(3 N-1)]^{T} \\
\boldsymbol{S} & =\operatorname{diag}\left\{\boldsymbol{S}_{\mathbf{1}}, \boldsymbol{S}_{\mathbf{2}}, \boldsymbol{S}_{\mathbf{3}}\right\}
\end{aligned}
$$

and $\boldsymbol{S}_{\boldsymbol{q}}$ is an $N \times K^{\prime}$ matrix with the $(n, k)$ th element given by $\left(n_{0}+n-k\right) . n_{0} \triangleq \frac{t_{0}}{T_{s}}$ and $K^{\prime}$ is the maximum number of sample-spaced channel taps. 
Let us consider the parameter vector as $\boldsymbol{\alpha}=\left\{v \psi \boldsymbol{h}^{T}\right\}$ where $v$ and $\psi$ are non-random parameters and $\boldsymbol{h}$ is a random parameter vector. Given $\boldsymbol{\alpha}$, the mean vector and the covariance matrix of the received training vector $\boldsymbol{r}$ are, respectively, given by $\boldsymbol{m}_{\boldsymbol{r}}=e^{j \psi} \boldsymbol{W}(v) \boldsymbol{S} \boldsymbol{h}$ and $\boldsymbol{C}_{\boldsymbol{r}}=\sigma_{n}^{2} \boldsymbol{I}$. The Bayesian version of the Fisher information matrix is given by [40]

$$
\boldsymbol{J}_{B}=\boldsymbol{J}_{D}+\boldsymbol{J}_{P}
$$

where the subscript $B$ refers to Bayesian, the subscript $D$ denotes the information due to the data and the subscript $P$ denotes the information due to the prior knowledge on the statistical property of $\boldsymbol{h}$. For the HCRB, we have

$$
\begin{aligned}
{\left[J_{D}\right]_{i, j}=} & E_{\boldsymbol{h}}\left[\operatorname{tr}\left[\boldsymbol{C}_{\boldsymbol{r}}^{-1} \frac{\partial \boldsymbol{C}_{\boldsymbol{r}}}{\partial \alpha_{i}} \boldsymbol{C}_{\boldsymbol{r}}{ }^{-1} \frac{\partial \boldsymbol{C}_{\boldsymbol{r}}}{\partial \alpha_{j}}\right]\right. \\
& \left.+2 \Re\left[\frac{\partial \boldsymbol{m}_{\boldsymbol{r}}{ }^{H}}{\partial \alpha_{i}} \boldsymbol{C}_{\boldsymbol{r}}{ }^{-1} \frac{\partial \boldsymbol{m}_{\boldsymbol{r}}}{\partial \alpha_{j}}\right]\right] \\
\boldsymbol{J}_{p}= & {\left[\begin{array}{ll}
\mathbf{0}_{2 \times 2} & \mathbf{0}_{2 \times 3 K^{\prime}} \\
\mathbf{0}_{3 K^{\prime} \times 2} \boldsymbol{Z}
\end{array}\right] }
\end{aligned}
$$

where $E_{\boldsymbol{h}}$ indicates the expectation with respect to $\boldsymbol{h}$, and $\boldsymbol{Z}=-E\left[\frac{\partial^{2} \ln p_{\boldsymbol{h}}(\boldsymbol{h})}{\partial \boldsymbol{h}^{2}}\right]$, where $p_{\boldsymbol{h}}(\boldsymbol{h})$ is the pdf of $\boldsymbol{h}$. Define $\boldsymbol{R}_{\boldsymbol{h}}=E\left[\boldsymbol{h} \boldsymbol{h}^{H}\right]$. Then, after straight-forward calculation, we obtain

$$
\begin{aligned}
{\left[J_{D}\right]_{1,1} } & =\frac{8 \pi^{2}}{N^{2} \sigma_{n}^{2}} \operatorname{tr}\left[\boldsymbol{S}^{\boldsymbol{H}} \Lambda^{2} \boldsymbol{S} \boldsymbol{R}_{\boldsymbol{h}}\right] \\
{\left[J_{D}\right]_{1,2} } & =\left[J_{D}\right]_{2,1}=\frac{4 \pi}{N \sigma_{n}^{2}} \operatorname{tr}\left[\boldsymbol{S}^{\boldsymbol{H}} \Lambda \boldsymbol{S} \boldsymbol{R}_{\boldsymbol{h}}\right] \\
{\left[J_{D}\right]_{2,2} } & =\frac{2}{\sigma_{n}^{2}} \operatorname{tr}\left[\boldsymbol{S}^{\boldsymbol{H}} \boldsymbol{S} \boldsymbol{R}_{\boldsymbol{h}}\right] \\
{\left[J_{D}\right]_{1, k \geq 3} } & =\left[J_{D}\right]_{k \geq 3,1}=\left[J_{D}\right]_{2, k \geq 3}=\left[J_{D}\right]_{k \geq 3,2}=0 .
\end{aligned}
$$

Then the HCRB for the OFO estimation in the MB-OFDM system is given by

$$
\mathrm{HCRB}=\left[J_{B}^{-1}\right]_{1,1}=\left(\left[J_{D}\right]_{1,1}-\left[J_{D}\right]_{1,2}\left[J_{D}\right]_{2,2}^{-1}\left[J_{D}\right]_{2,1}\right)^{-1} .
$$

We obtain $\boldsymbol{R}_{\boldsymbol{h}}$ from simulation when calculating the HCRB numerically.

\section{REFERENCES}

[1] M. Z. Win and R. A. Scholtz, "Impulse radio: how it works," IEEE Commun. Lett., vol. 2, no. 2, pp. 36-38, Feb. 1998.

[2] M. Z. Win and R. A. Scholtz, "Ultra-wide bandwidth time-hopping spread-spectrum impulse radio for wireless multiple-access communications," IEEE Trans. Commun., vol. 48, no. 4, pp. 679-691, Apr. 2000.

[3] T. Q. S. Quek and M. Z. Win, "Analysis of UWB transmitted reference communication systems in dense multipath channels," IEEE J. Select. Areas Commun., vol. 23, no. 9, pp. 1863-1874, Sept. 2005.

[4] A. Giorgetti, M. Chiani, and M. Z. Win, "The effect of narrowband interference on wideband wireless communication systems," IEEE Trans. Commun., vol. 53, no. 12, pp. 2139-2149, Dec. 2005.

[5] M. Z. Win, "A unified spectral analysis of generalized time-hopping spread-spectrum signals in the presence of timing jitter," IEEE J. Select. Areas Commun., vol. 20, no. 9, pp. 1664-1676, Dec. 2002.

[6] A. Ridolfi and M. Z. Win, "Ultrawide bandwidth signals as shot-noise: a unifying approach," IEEE J. Select. Areas Commun., vol. 24, no. 4, pp. 899-905, Apr. 2006.

[7] A. Batra, et. al., "Multi-band OFDM physical layer proposal for IEEE 802.15 task group 3a," IEEE P802.15-03/268r3, Orlando, FL, USA, Mar. 2004.

[8] IEEE 802.15 working group for wireless personal area networks (WPANs)' "Multi-band OFDM physical layer proposal for IEEE 802.15 task group 3a," Mar. 2004.

[9] Standard ECMA-368,"High rate ultra wideband PHY and MAC standard," Dec. 2005

[10] L. J. Cimini, "Analysis and simulation of a digital mobile channel using orthogonal frequency division multiplexing," IEEE Trans. Commun., vol. 33, pp. 665-675, July 1985.
[11] J. A. C. Bingham, "Multicarrier modulation: an idea whose time has come," IEEE Commun. Mag., vol. 28, no. 5, pp. 5-14, May 1990.

[12] D. Dardari and V. Tralli, "High-speed indoor wireless communications at $60 \mathrm{GHz}$ with coded OFDM," IEEE Trans. Commun., vol. 47, no. 11, pp. 1709-1721, Nov. 1999.

[13] D. Dardari, M. G. Martini, M. Mazzotti, and M. Chiani, "Layered video transmission on adaptive OFDM wireless systems," Eurasip J. Applied Signal Processing, Hindawi Publishing Corp., vol. 10, pp. 1557-1567, 2004.

[14] D. Dardari, "Ordered subcarrier selection algorithm for OFDM based high-speed WLANs," IEEE Trans. Wireless Commun., vol. 3, no. 5, pp. 1452-1458, Sept. 2004.

[15] D. Dardali, V. Tralli, and A. Vaccari, "A theoretical characterization of nonlinear distortion effects in OFDM systems," IEEE Trans. Commun., vol. 48, no. 10, pp. 1755-1764, Oct. 2000.

[16] T. Pollet, M. Van Bladel, and M. Moeneclaey, "BER sensitivity of OFDM systems to carrier frequency offset and Wiener phase noise," IEEE Trans. Commun., vol. 43, pp. 191-193, Feb./Mar./Apr. 1995.

[17] M. Gudmundson and P. O. Anderson, "Adjacent channel interference in an OFDM system," in Proc. IEEE Vehicular Technol. Conf., Atlanta, GA, pp. 918-922, May 1996.

[18] P. Robertson and S. Kaiser, "The effects of Doppler spreads in OFDM(A) mobile radio systems," in Proc. IEEE Vehicular Technol. Conf. (Fall), vol. 1, pp. 329-333, Sept. 1999.

[19] H. Steendam and M. Moeneclaey, "Synchronization sensitivity of multicarrier systems," European Commun., ETT special issue on multi-carrier spread spectrum, vol. 52, no. 5, pp. 834-844, May 2004.

[20] P. H. Moose, "A technique for orthogonal frequency division multiplexing frequency offset correction," IEEE Trans. Commun., pp. 2908-2914, Oct. 1994.

[21] T. M. Schmidl and D. C. Cox, "Robust frequency and timing synchronization for OFDM," IEEE Trans. Commun., pp. 1614-1621, Dec. 1997.

[22] M. Morelli and U. Mengali, "An improved frequency offset estimator for OFDM applications,” IEEE Commun. Lett., pp. 75-77, Mar. 1999.

[23] T. Keller, L. Piazzo, P. Mandarini, and L. Hanzo, "Orthogonal frequency division multiplex synchronization techniques for frequency-selective fading channels," IEEE J. Select. Areas Commun., vol. 19, pp. 999-1008, June 2001.

[24] H. Minn, P. Tarasak, and V. K. Bhargava, "OFDM frequency offset estimation based on BLUE principle," in Proc. IEEE VTC 2002 (Fall), pp. 1230-1234.

[25] H. Minn, P. Tarasak, and V. K. Bhargava, "Some issues of complexity and training symbol design for OFDM frequency offset estimation methods based on BLUE principle," in Proc. IEEE VTC 2003 (Spring), pp. 1288-1292.

[26] H. Minn and P. Tarasak, "Improved maximum likelihood frequency offset estimation based on likelihood metric design," IEEE Trans. Signal Processing, vol. 54, no. 6, pp. 2076-2086, June 2006.

[27] M. Z. Win and R. A. Scholtz, "On the robustness of ultra-wide bandwidth signals in dense multipath environments," IEEE Commun. Lett., vol. 2, no. 2, pp. 51-53, Feb. 1998.

[28] M. Z. Win and R. A. Scholtz, "On the energy capture of ultra-wide bandwidth signals in dense multipath environments," IEEE Commun. Lett., vol. 2, no. 9, pp. 245-247, Sept. 1998.

[29] R. J. Cramer, R. A. Scholtz, and M. Z. Win, "An evaluation of the ultrawideband propagation channel," IEEE Trans. Antennas Propag., vol. 50, no. 5, pp. 561-570, May 2002.

[30] D. Cassioli, M. Z. Win, and A. F. Molisch, "The ultra-wide bandwidth indoor channel: from statistical model to simulations," IEEE J. Select. Areas Commun., vol. 20, no. 6, pp. 1247-1257, Aug. 2002.

[31] M. Z. Win and R. A. Scholtz, "Characterization of ultra-wide bandwidth wireless indoor communications channel: a communication theoretic view," IEEE J. Select. Areas Commun., vol. 20, no. 9, pp. 1613-1627, Dec. 2002.

[32] C.-C. Chong and S. K. Yong, "A generic statistical based UWB channel model for high-rise apartments," IEEE Trans. Antennas Propag., vol. 53, no. 8, pp. 2389-2399, Aug. 2005.

[33] C.-C. Chong, Y. Kim, S. K. Yong, and S. S. Lee, "Statistical characterization of the UWB propagation channel in indoor residential environment," Wiley J. Wireless Commun. and Mobile Computing, vol. 5, no. 5, pp. 503-512, Aug. 2005.

[34] A. F. Molisch, J. R. Foerster, and M. Pendergrass, "Channel models for ultrawideband personal area networks," IEEE Wireless Commun. Mag., vol. 10, no. 6, pp. 14-21, Dec. 2003.

[35] IEEE 802.15 Working Group for Wireless Personal Area Networks (WPANs)' "Time variance for UWB wireless channels," Nov. 2002. 
[36] Y. Li, T. Jacobs, and H. Minn, "Frequency offset estimation for MBOFDM-based UWB systems," in Proc. IEEE ICC 2006, Istanbul, Turkey, June 2006.

[37] C.-C. Chong, C.-M. Tan, D. I. Laurenson, S. McLaughlin, M. A. Beach, and A. R. Nix, "A novel wideband dynamic directional indoor channel model based on a Markov process," IEEE Trans. Wireless Commun., vol. 4, no. 4, pp. 1539-1552, July 2005.

[38] A. Batra, J. Balakrishnan, G. R. Aiello, J. R. Foerster, and A. Dabak, "Design of a multiband OFDM system for realistic UWB channel environments," IEEE Trans. Microwave Theory and Techniques, pp. 21232137, Sept. 2004.

[39] S. M. Kay, Fundamentals of Statistical Signal Proczessing: Estimation Theory. Prentice Hall PTR, 1993.

[40] H. L. Van Trees, Optimum Array Processing. New York: Wiley Interscience, 2002.

[41] F. A. Graybill, Introduction to Matrices with Applications in Statistics. Wadsworth Publishing Company, Inc., Belmont, CA, 1969.

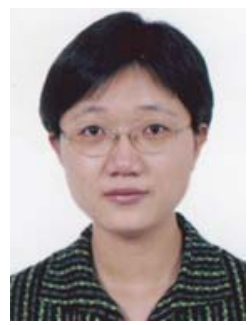

Yinghui Li (S'05) received the B.E. and M.S degrees in Electrical Engineering from the Nanjing University of Aeronautics and Astronautics, Nanjing, China, in 2000 and 2003, respectively. She received the Ph.D. degree in Electrical Engineering from the University of Texas at Dallas in 2007. Her research interests are in the applications of statistical signal processing and training signal designs in synchronization, channel estimation and detection problems in broadband wireless communications.

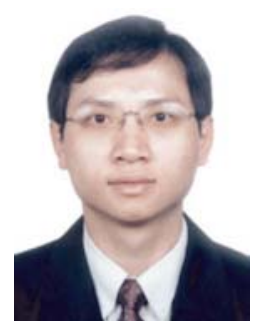

Hlaing Minn (S'99-M'01-SM'07) received his B.E degree in Electronics from Yangon Institute of Technology, Yangon, Myanmar, in 1995, M.Eng. degree in Telecommunications from Asian Institute of Technology (AIT), Pathumthani, Thailand, in 1997 and $\mathrm{Ph} . \mathrm{D}$. degree in Electrical Engineering from the University of Victoria, Victoria, BC, Canada, in 2001.

He was with the Telecommunications Program in AIT as a laboratory supervisor during 1998 . He was a research assistant from 1999 to 2001 and a postdoctoral research fellow during 2002 in the Department of Electrical and Computer Engineering at the University of Victoria. Since September 2002, he has been with the Erik Jonsson School of Engineering and Computer Science, the University of Texas at Dallas, USA, as an Assistant Professor. His research interests include wireless communications, statistical signal processing, error control, detection, estimation, synchronization, signal design, cross-layer design, and cognitive radios. He is an Editor for the IEEE Transactions on Communications.

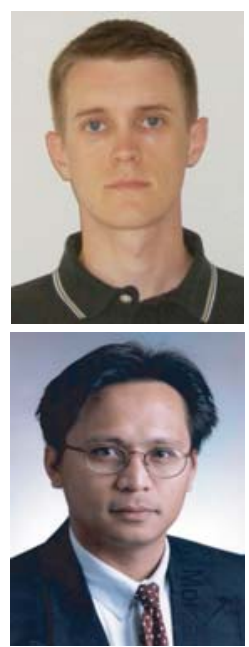

Trent Jacobs (S'07) received his B.S. in Electrical Engineering from the University of Texas at Dallas in 2005. Subsequently, he received his M.S. degree in Electrical Engineering, also from the University of Texas at Dallas, in 2007. His research area encompasses frequency offset estimation, synchronization, and reduced complexity methods for ultrawideband and wireless sensor networks.

Moe Z. Win (S'85-M'87-SM'97-F'04) received the B.S. degree (magna cum laude) from Texas A\&M University, College Station, in 1987 and the M.S. degree from the University of Southern California (USC), Los Angeles, in 1989, both in Electrical Engineering. As a Presidential Fellow at USC, he received both an M.S. degree in Applied Mathematics and the Ph.D. degree in Electrical Engineering in 1998 .

Dr. Win is an Associate Professor at the Laboratory for Information \& Decision Systems (LIDS), Massachusetts Institute of Technology (MIT). Prior to joining MIT, he spent five years at AT\&T Research Laboratories and seven years at the Jet Propulsion Laboratory. His main research interests are the applications of mathematical and statistical theories to communication, detection, and estimation problems. Specific current research topics include measurement and modeling of time-varying channels, design and analysis of multiple antenna systems, ultra-wide bandwidth (UWB) systems, optical transmission systems, and space communications systems. Professor Win has been actively involved in organizing and chairing a number of international conferences. He served as the Technical Program Chair for the IEEE Conference on Ultra Wideband in 2006, the IEEE Communication Theory Symposia of ICC-2004 and Globecom-2000, and the IEEE Conference on Ultra Wideband Systems and Technologies in 2002; Technical Program Vice-Chair for the IEEE International Conference on Communications in 2002; and the Tutorial Chair for the IEEE Semiannual International Vehicular Technology Conference in Fall 2001. He served as a chair (2004-2006) and secretary (2002-2004) for the Radio Communications Committee of the IEEE Communications Society. Dr. Win is currently an Editor for IEEE Transactions on Wireless Communications. He served as Area Editor for Modulation and Signal Design (2003-2006), Editor for Wideband Wireless and Diversity (20032006), and Editor for Equalization and Diversity (1998-2003), all for the IEEE Transactions on Communications. He was Guest-Editor for the 2002 IEEE Journal on Selected Areas in Communications (Special Issue on UltraWideband Radio in Multiaccess Wireless Communications). Professor Win received the International Telecommunications Innovation Award from Korea Electronics Technology Institute in 2002, a Young Investigator Award from the Office of Naval Research in 2003, and the IEEE Antennas and Propagation Society Sergei A. Schelkunoff Transactions Prize Paper Award in 2003. In 2004, Dr. Win was named Young Aerospace Engineer of the Year by AIAA, and garnered the Fulbright Foundation Senior Scholar Lecturing and Research Fellowship, the Institute of Advanced Study Natural Sciences and Technology Fellowship, the Outstanding International Collaboration Award from the Industrial Technology Research Institute of Taiwan, and the Presidential Early Career Award for Scientists and Engineers from the United States White House. He was honored with the 2006 IEEE Eric E. Sumner Award "for pioneering contributions to ultra-wide band communications science and technology." Professor Win is an IEEE Distinguished Lecturer and elected Fellow of the IEEE, cited "for contributions to wideband wireless transmission." 